Jan Philipp Schägner, Luke Brander, Joachim Maes, Volkmar Hartje

\title{
Mapping ecosystem services' values: Current practice and future prospects
}

Journal article | Accepted manuscript (Postprint)

This version is available at https://doi.org/10.14279/depositonce-8670

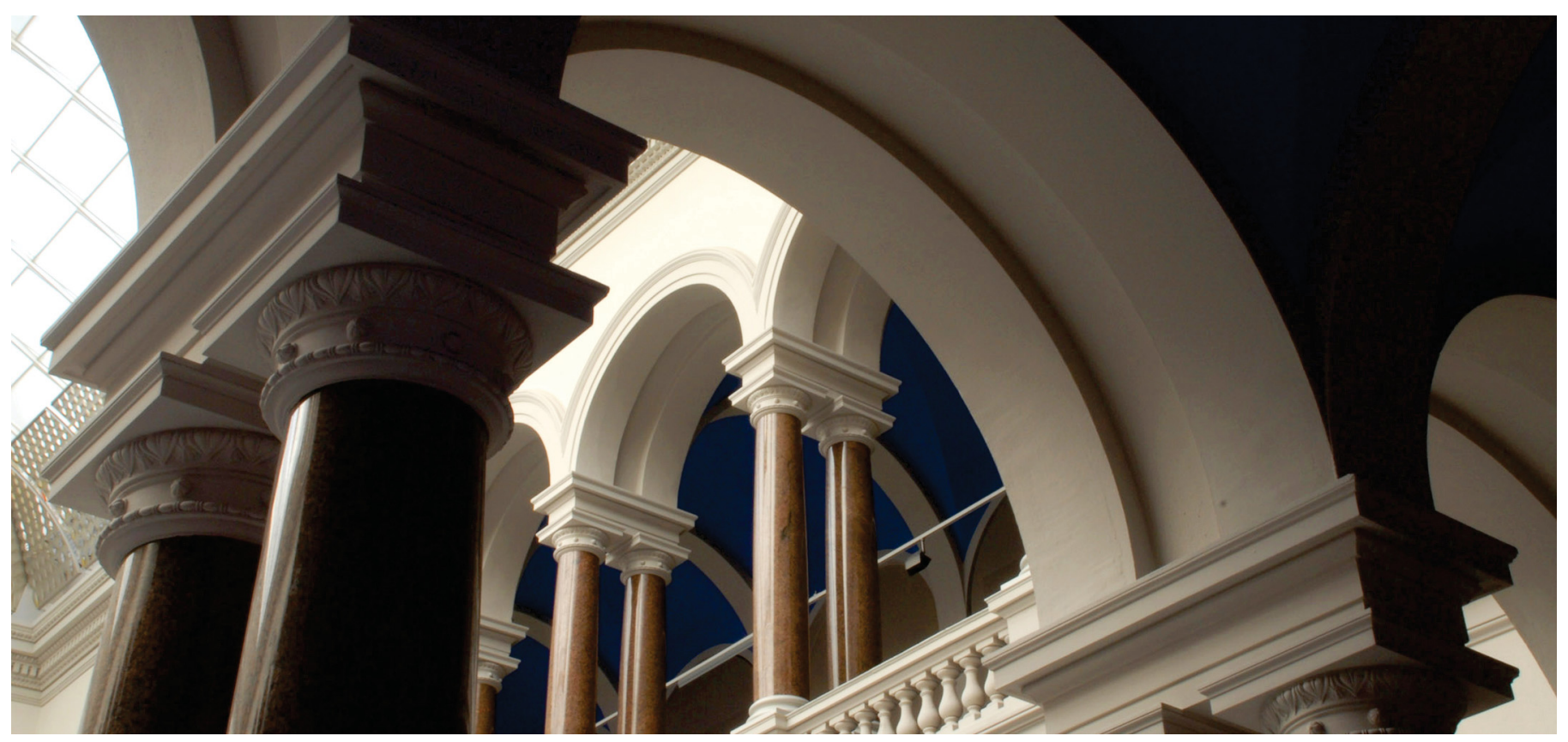

Schägner, J. P., Brander, L., Maes, J., \& Hartje, V. (2013). Mapping ecosystem services' values: Current practice and future prospects. Ecosystem Services, 4, 33-46.

https://doi.org/10.1016/j.ecoser.2013.02.003 


\title{
Mapping Ecosystem Services' Values: Current Practice and Future Prospects
}

Jan Philipp Schägner ${ }^{\mathrm{a}}$, Luke Brander ${ }^{\mathrm{b}}$, Joachim Maes ${ }^{\mathrm{a}}$, Volkmar Hartje

\section{Keywords:}

Ecosystem service assessment

Ecosystem service mapping

Ecosystem service valuation

Ecosystem service modelling

Value transfer

Land use policy assessment

\begin{abstract}
:
Mapping of ecosystem services' (ESS) values means valuing ESS in monetary terms across a relatively large geographical area and assessing how values vary across space. Thereby, mapping of ESS values reveals additional information as compared to traditional site-specific ESS valuation, which is beneficial for designing land use policies for maintaining ESS supply. Since the well-known article by Costanza et al. (1997), who mapped global ESS values, the number of publications mapping ESS values has grown exponentially, with almost $60 \%$ being published after 2007 . Within this paper, we analyse and review articles that map ESS values. Our findings show that methodologies, in particular how spatial variations of ESS values are estimated, their spatial scope, rational and ESS focus differ widely. Still, most case studies rely on relatively simplistic approaches using land use/cover data as a proxy for ESS supply and its values. However, a tendency exists towards more sophisticated methodologies using the ESS models and value functions, which integrate a variety of spatial variables and which are validated against primary data. Based on our findings, we identify current practices and developments in the mapping of EES values and provide guidelines and recommendations for future applications and research.
\end{abstract}

aEuropean Commission, Joint Research Centre, Ispra, Italy; ${ }^{b}$ Vrije Universiteit, Amsterdam, The Netherlands; 'Technical University Berlin, Germany

Published in: 2013. Ecosystem Services, Special Issue on Mapping and Modelling Ecosystem Services, 4 (June): 33-46. doi.10.1016/i.ecoser.2013.02.003. 


\section{Introduction}

The framework of ecosystem services (ESS) is widely used for communicating links between ecosystems and human well-being (MA 2005). Manifold studies aim to integrate ESS assessments into decision making processes (TEEB 2010; UK NEA 2011). The economic value (i.e., contribution to human welfare) of an ESS is, as with any good or service, determined by its supply and demand. The supply side of an ESS is largely determined by ecological processes and characteristics (e.g., functioning, fragmentation, productivity, resilience or climate) that may be influenced by human activities, either deliberately or inadvertently. The understanding and modelling of the supply of ESS has largely been taken up by natural scientists (e.g., ecologists, geographers, hydrologists). The demand side is largely determined by the characteristics of human beneficiaries of the ESS (population, preferences, distance to resource etc.). The understanding and modelling of the demand side has largely been taken up by economists. It has been recognised that the determinants of both, the supply and demand of ESS, are spatially variable, which makes the assessment of ESS values inherently spatial. In recent years, a growing body of literature assesses ESS spatially by producing digital maps either of ESS supply or its value. In particular, the mapping of monetary values for ESS value has become an active research topic in recent years (Troy and Wilson 2006; Maes et al. 2011a). In this paper we review studies that map monetary values of ESS. We define mapping of ESS values as the valuation of ESS in monetary terms across a relatively large geographical area that includes the examination of how values vary across space. ${ }^{1}$ Thereby, mapping of ESS values reveals additional information as compared to traditional sitespecific ESS valuation, which is beneficial for designing efficient policies and institutions for maintaining ESS supply.

To some extent spatial issues have been disregarded in environmental and resource economics, including ESS valuation, but have attracted increasing attention with the emergence of advanced GIS technology in the 90's (Bockstael 1996). The first studies to map ESS values examine recreational values for Welsh forests (Bateman et al. 1995) and multiple ESS across a protected area in Belize (Eade and Moran 1996). A milestone in this development is the well-known paper by Costanza et al. (1997), in which global ESS values are mapped. This paper raised a lot of attention and initiated a debate on value mapping in general and on the meaningfulness of aggregate global values. Since then, the number of publications mapping ESS values has grown exponentially, with almost $60 \%$ being published after 2007 (see Figure 1). The methodologies applied in these studies differ widely, in particular with respect to how spatial variation in ESS values is estimated. The precision and accuracy of mapped ESS values has been questioned, and accordingly the utility for policy guidance. However, no consensus has been reached on which methods can and should be used to inform specific policy contexts (De Groot et al. 2010). Until now, no comprehensive review of the literature on mapping ESS values has been conducted.

Within this paper, we review all peer reviewed journal articles published before 2012 that map monetary ESS values. Articles were obtained by searching the Scopus, Science Direct and Google scholar databases with various key word combinations and by scanning the references of all relevant papers. In total, we obtained 384 articles of which 143 map ESS. We excluded all studies from the review that map only ESS supply (54) and that map non-monetary ESS values (20), because nonmonetary valuation follows a different theoretical framework and applies a different set of valuation techniques. We analysed the remaining 69 articles and reviewed them according to the methodologies used for ESS quantification and valuation, the ESS assessed, study rationale and case study area

\footnotetext{
${ }^{1}$ The literature that we examine does therefore not only include studies that produce graphical value maps but also includes analyses that explicitly address spatial variability in values.
} 
characteristics. The purpose of this review is to identify current practices and developments in the mapping of monetary EES values with a view to providing recommendations for future applications and research.

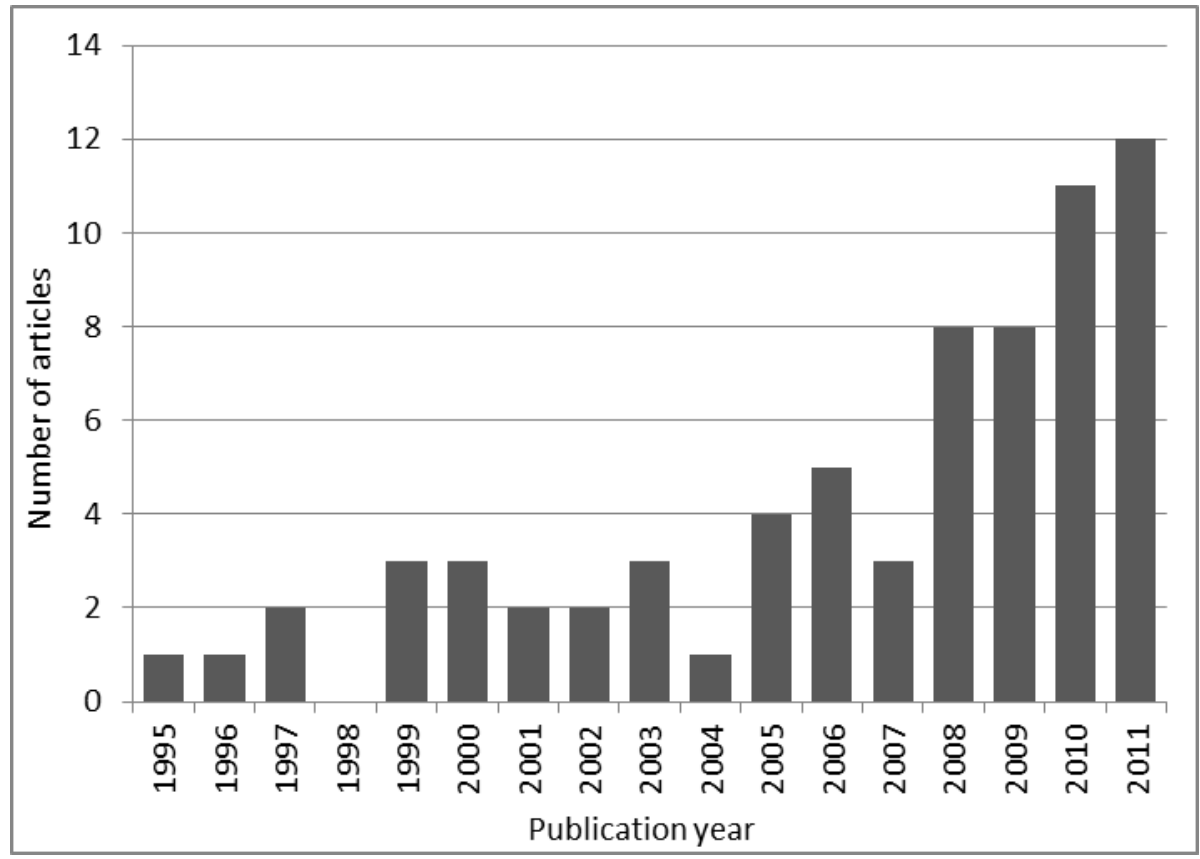

Figure 1: Published articles per year.

The paper is organised as follows: in Section 2 we give an overview of the rationale and contribution of ESS value mapping to ESS research and policy making. Section 3 gives a quantitative review of general study characteristics, such as location, scale of analysis, and ecosystems and ESS addressed. In Section 4, different methodologies used for mapping ESS values are analysed and studies are classified within a methodology matrix. We discuss evidence on the accuracy of current value mapping exercises and evaluate the different methodologies. In Section 5, we give an outlook on future prospects and avenues for development. Finally, Section 6 provides some conclusions.

\section{Why Map Values?}

Natural ecosystems produce various ESS, which strongly contribute to human well-being (TEEB 2010; MA 2005). Nevertheless, due to the public good characteristics of many ecosystems and their vulnerability to externalities, such as air, soil and water contamination, the costs of ecosystem degradation are not sufficiently incorporated into individual or public decision-making. As a result, ecosystems in all parts of the world are being degraded to a suboptimal extent, causing loss of ESS supply. Various national and supranational policies have been introduced to protect natural ecosystems, which have only been partially effective (e.g. Ramsar Convention on wetlands of international importance; Convention on Biological Diversity 2010 target). Reversing the degradation of ecosystems requires "significant changes in policies, institutions, and practices that are not currently under way" (MA 2005).

One of the main challenges in designing effective policies derives from the complexity of integrating multidimensional environmental impacts into decision making processes. Typically, decisions are based mainly on information that is well understood and known with high certainty, for example information on readily observable financial or market transactions. Ecological externalities are typically insufficiently considered because of uncertain estimates regarding expected impacts, difficulties in interpreting results from various disciplines and difficulties in translating impacts into changes in social 
welfare. Monetary valuation of ESS is a method to overcome such difficulties. It enables the aggregation of multidimensional costs and benefits of alternative measures within a one-dimensional welfare measure (Pearce et al. 2006). Although the practice of monetary valuation and its underlying framework are subject to debate and criticism (Spash and Carter 2001; Sagoff 2004), the concept of monetary valuation and cost-benefit analysis is widely accepted and subject to intensive research activity.

The estimation of accurate ESS values, however, is not straightforward, in part due to spatial heterogeneity in biophysical and socioeconomic conditions. The spatial perspective of variation in ESS values is relatively new and has not been extensively researched. Insufficient knowledge exists about how ESS values differ across space and what their spatial determinants are (Bockstael 1996; Bateman et al. 2002; Plummer 2009; De Groot et al. 2010). With the development of advanced GIS technology, mapping of ESS values has emerged and become an important research issue in recent years.

As compared to traditional site-specific ESS valuation, mapping reveals additional valuable information. Besides communication and visualisation, it makes site specific ESS values available on a large spatial scale. Thereby, it allows policy makers to extract estimated values easily from a database at any scale and for any site of interest in order to evaluate potential policy measures. Time consuming primary valuation or value transfer studies may not be necessary. Thereby, spatially explicit ESS value maps have specific advantages for several policy applications including: (1) green accounting, (2) land use policy evaluation, (3) resource allocation and (4) payments for ESS. Figure 2 presents the frequency with which specific policy applications are mentioned as the potential end-use of value data in the ESS mapping literature.

(1) Green accounting: mapping of ESS values allows for estimating a green Gross Domestic Product (GDP) at different spatial scales, by summing up total ESS values across the region of interest (TEEB 2010). (2) Land use policy evaluation: mapping of ESS values allows for the evaluation of broad land use policies at a regional or even supranational level. Typically, land uses are multifunctional and therefore provide multiple services. ESS value mapping displays trade-offs and synergies in ESS values, which may result from land use change. (3) Resource allocation: mapping of ESS values not only supports decisions on whether or not to conduct a policy measure, it also indicates where to conduct a policy measure. It allows the identification of locations in order to minimise negative or maximise positive ecological side effects. For example, by identifying ESS hot spots for conservation it allows the assessment of "synergies and trade-offs in conserving biodiversity and ecosystem services" (Naidoo et al. 2008). (4) Payments for ESS: by making ESS values spatially explicit, schemes of payments for ESS can be designed to allow for more efficient incentives across providers of ESS. 


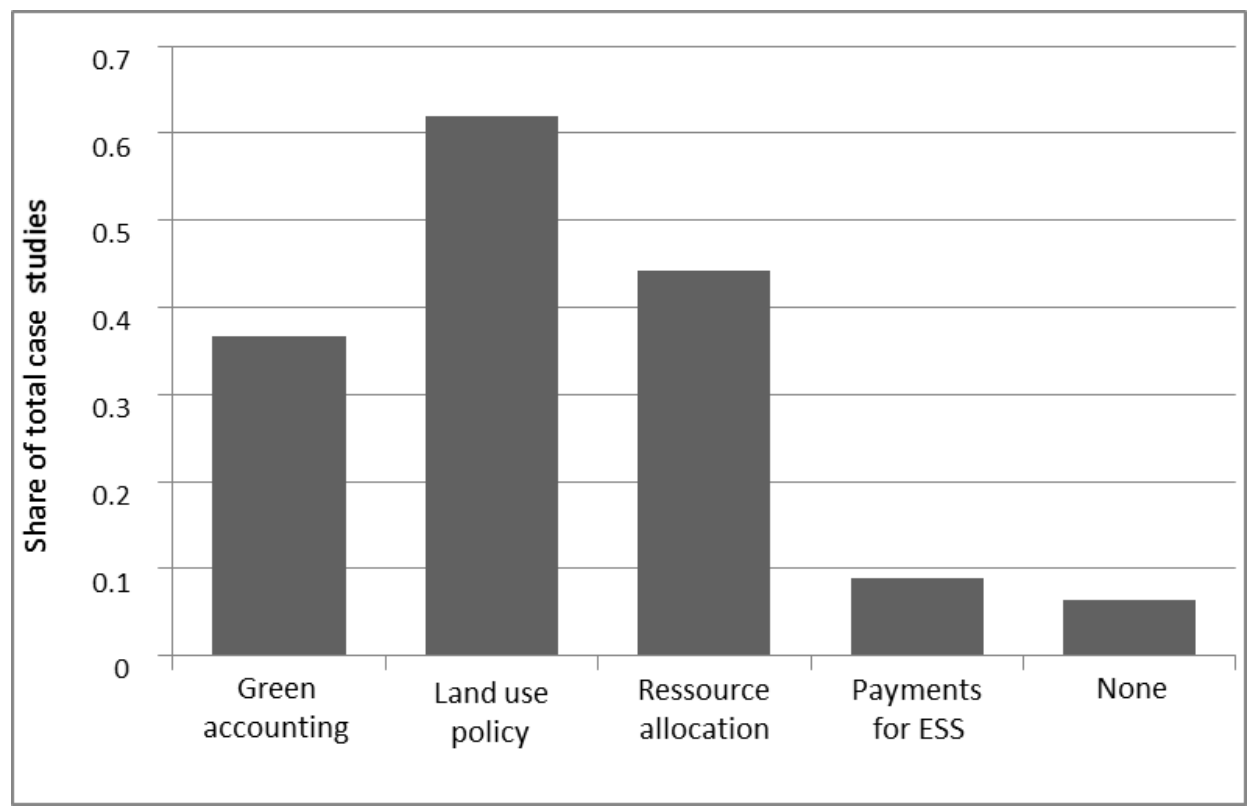

Figure 2: Citation of policy applications in ESS mapping literature.

\section{Quantitative Review of Studies Mapping ESS Values}

In total we analysed 69 publications, which include 79 separate case studies. Studies differ strongly with respect to their spatial scope, the ecosystems and ESS assessed and the methodologies applied. Case study areas are mainly located in three continents, with 34\% in Europe (mainly UK), 24\% in North America (mainly USA) and $22 \%$ in Asia (mainly China). Figure 3 shows the spatial distribution of the case studies across the world. The colour indicates the number of studies covering each country. The minimum for each country is five as there are five global case studies. The continental, national and subnational case studies are then added for each country.

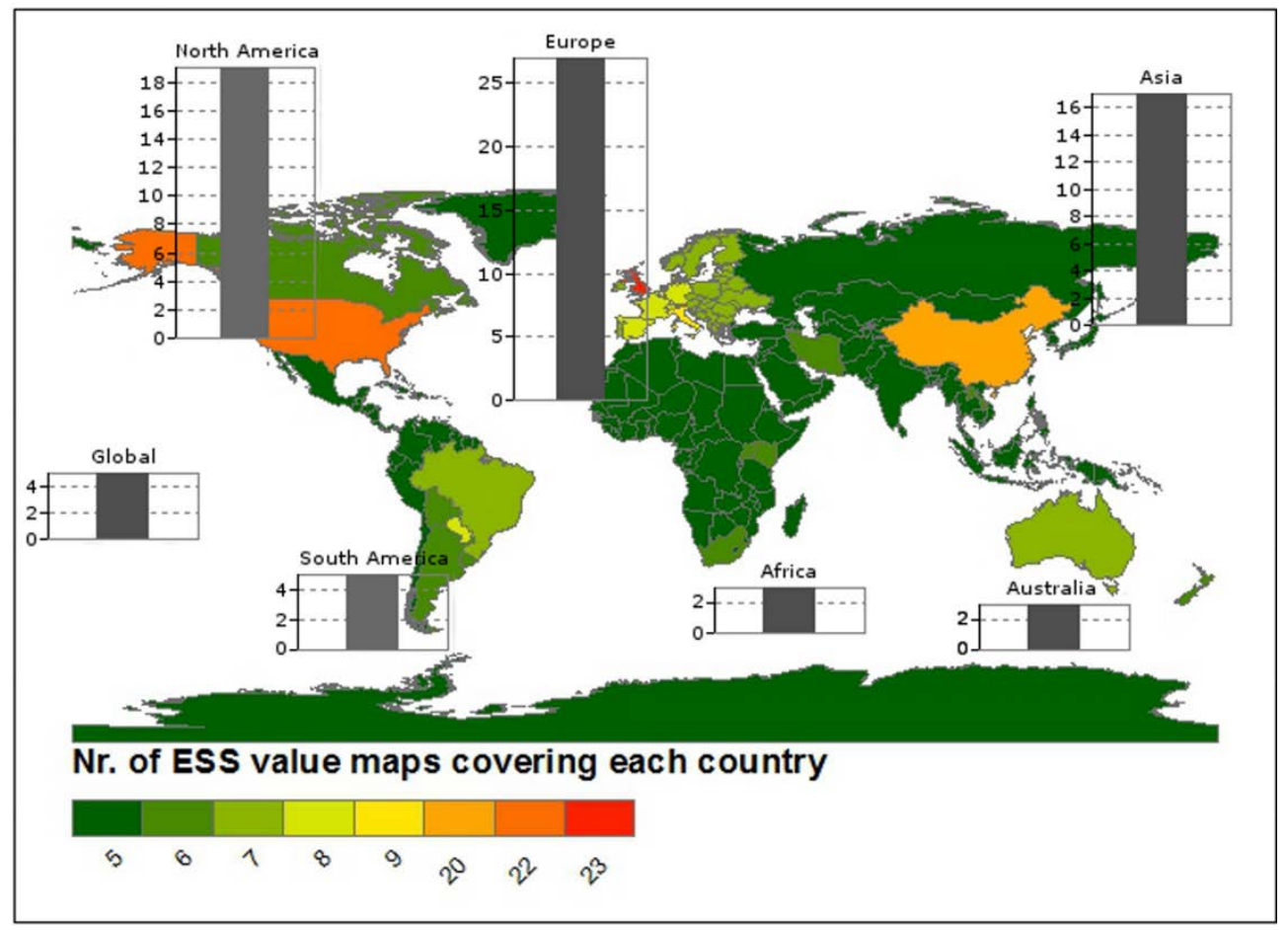

Figure 3: Spatial distribution of case study areas. 
Study areas differ in size, ranging from global to local assessments (see Figure 4), with the smallest case study area comprising a 550 ha forest in the surrounding of Gent, Belgium (Moons et al. 2008). Approximately $20 \%$ of all studies are 'local' applications with a case study area smaller than $1000 \mathrm{~km}^{2}$. Typically, they focus on a single protected area, a single forest or an urban area. Approximately $23 \%$ focus on case study areas between $1000 \mathrm{~km}^{2}$ and $10,000 \mathrm{~km}^{2}$. Most of them are defined by the borders of an administrative region. Study site areas from $10,000 \mathrm{~km}^{2}$ up to $100,000 \mathrm{~km}^{2}$ comprise $24 \%$ of all studies. They contain mainly regional to national assessments. Approximately another $24 \%$ of all study areas are continental, supra national or global ESS value assessments with study areas above 100,000 $\mathrm{km}^{2}$.

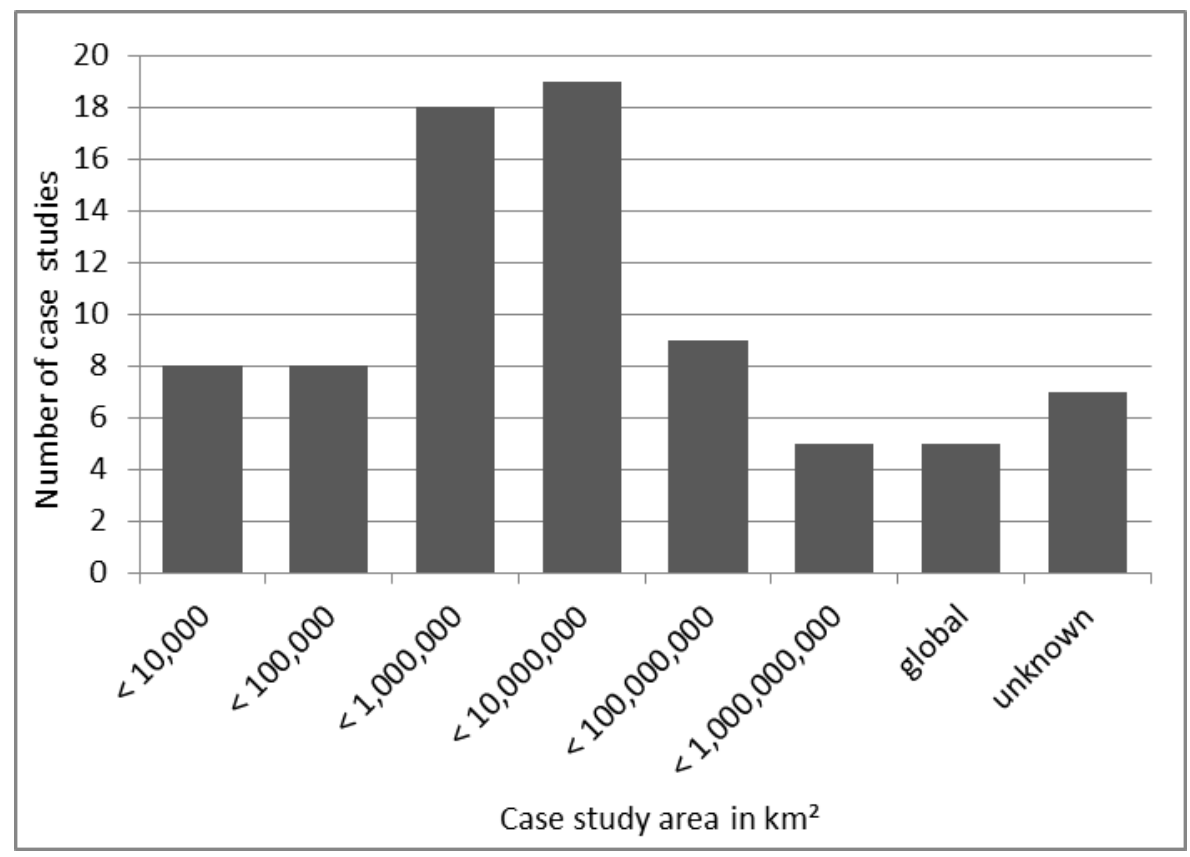

Figure 4: Study site area size.

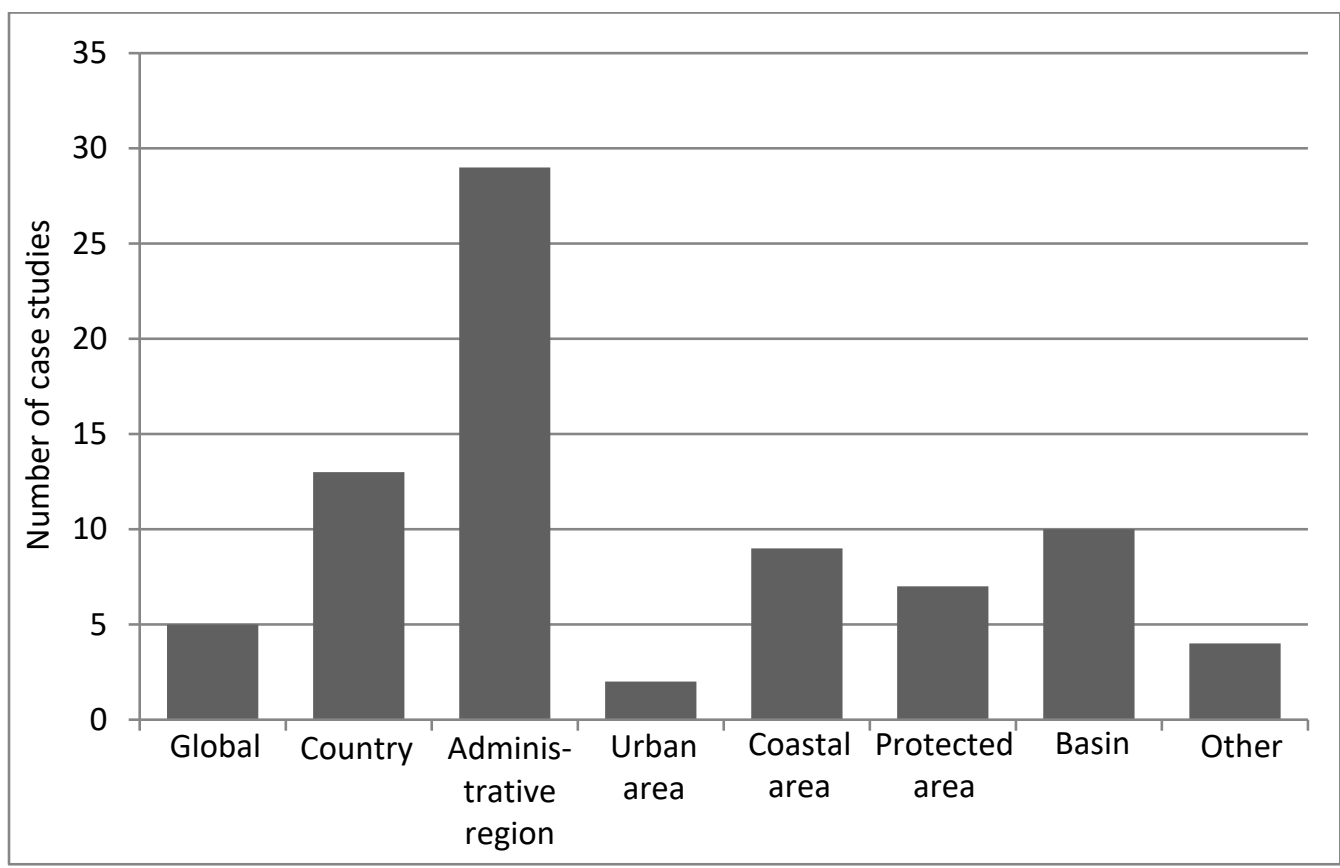

Figure 5: Types of study areas. 
Most study area definitions depend on political boarders, such as administrative regions (37), countries $(16 \%)$, urban areas $(3 \%)$ or protected areas $(9 \%)$. Study areas defined by some geomorphological features are mainly related to river features $(13 \%)$ such as basins or watersheds or are coastal areas (11\%), such as a bay or an estuary (see Figure 5 ).

Most studies focus on more than four (multiple) land cover or land use classes (LCLU) (see Figure 6), which is expected given that values are generally mapped across larger areas. Some smaller case studies, however, focus on specific landscapes involving only one to four LCLU. Some studies map values of only one land cover within a larger area, for example all forests in Wales (Bateman et al. 1999a).

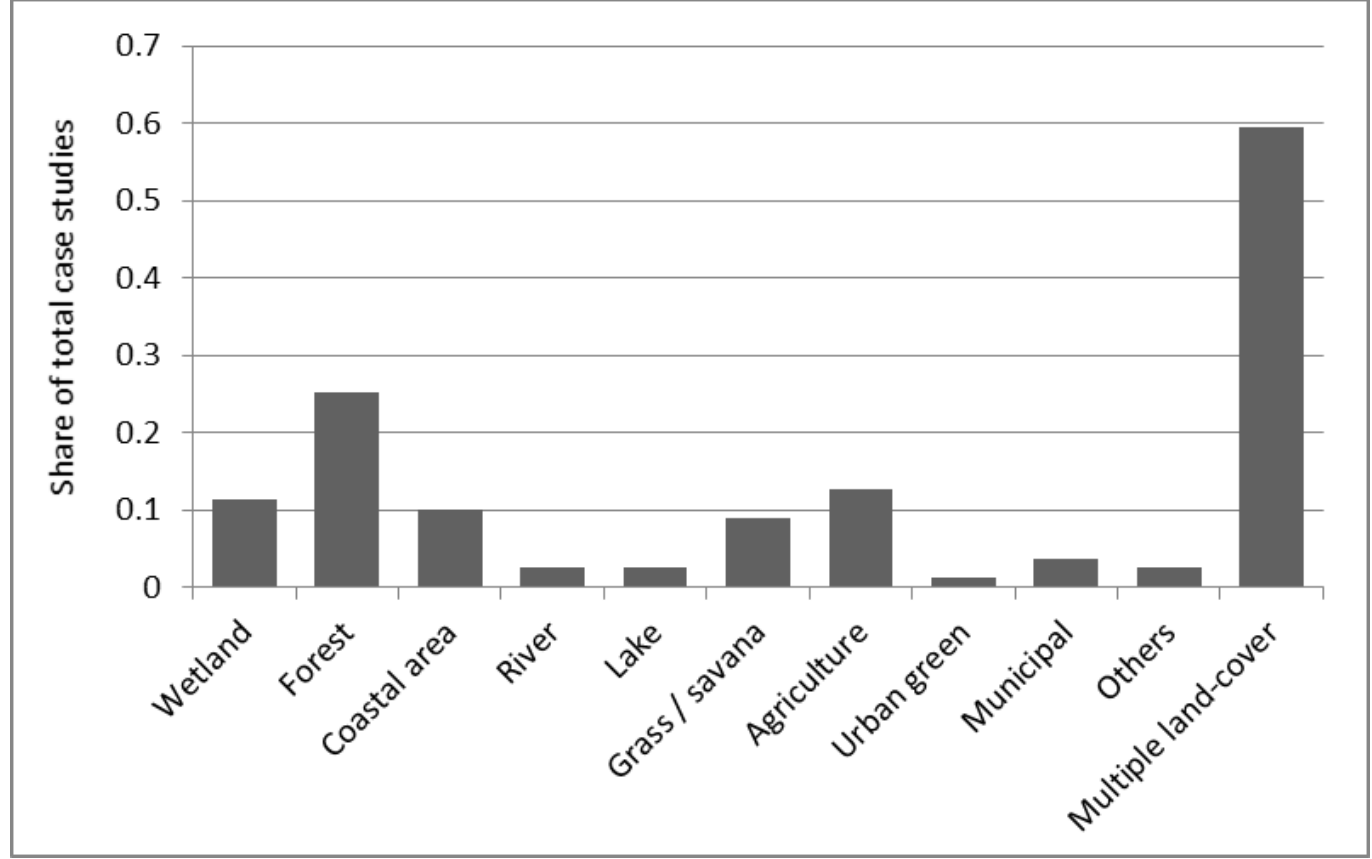

\section{Figure 6: Ecosystems assessed.}

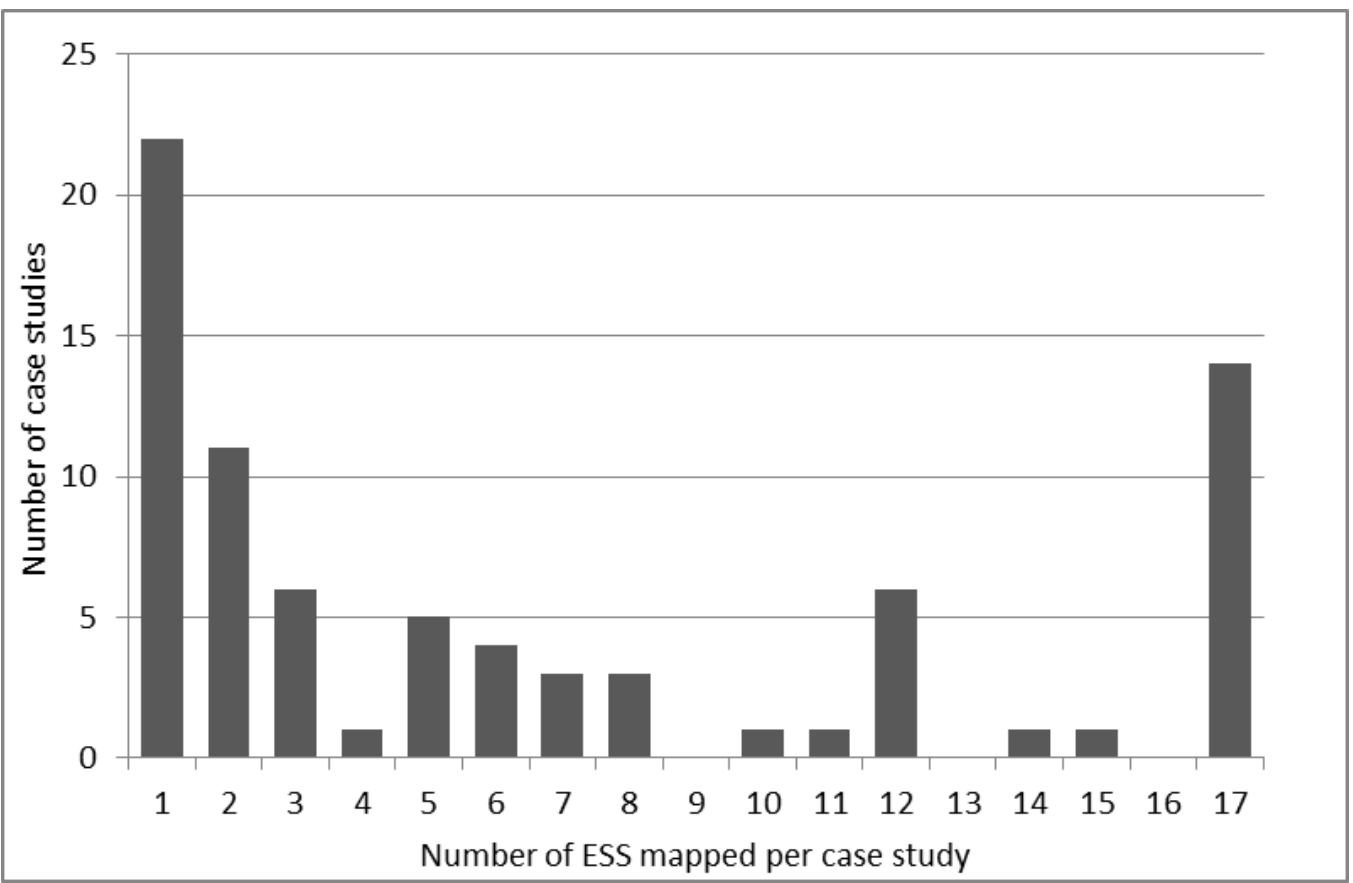

Figure 7: Number of ESS mapped per case study. 
On average, each study maps values for seven ESS. However, many studies focus only on one single ESS (28\%) and about $50 \%$ map three or less ESS. At the other end of the scale, $18 \%$ of all studies follow the approach of Costanza et al. (1997) and accordingly map 17 ESS (see Figure 7).

The set of ESS mapped by Costanza et al. (1997) are mapped frequently, as their approach has been replicated several times. In total, recreation is the most frequently mapped ESS with 50 case studies, followed by the control of greenhouse gases (mainly carbon sequestration). The frequency with which each ESS has been mapped is shown in Figure 8.

Many studies do not give any information on the resolution at which values are mapped. For studies that do provide such information, the range is from $1 \mathrm{~m}$ to $10,000 \mathrm{~m}$ resolution (see Figure 9).

\section{Methodologies for Mapping ESS Values}

ESS valuation applications involve two dimensions: (1) a biophysical assessment of ESS supply and (2) a socioeconomic assessment of the value per unit of ESS. If ESS values are mapped, variations in ESS values across space are either assessed by mapping spatial variations of ESS supply, by mapping spatial variations of the value per unit of ESS or by a combination of both dimensions.

In the reviewed literature, we identified five different methodologies used for mapping ESS supply (Eigenbrod et al. 2010b) and, in analogy to environmental value transfer, four different methodologies of attaching a value per unit ESS. In this section we first describe these different methodologies used for assessing ESS supply and its value. We then give an overview and examples of how these methodologies are used in combination in order to map ESS values. Thereafter, we discuss evidence on the accuracy and precision of ESS value maps. Based on our findings we then discuss and evaluate the different methodologies.

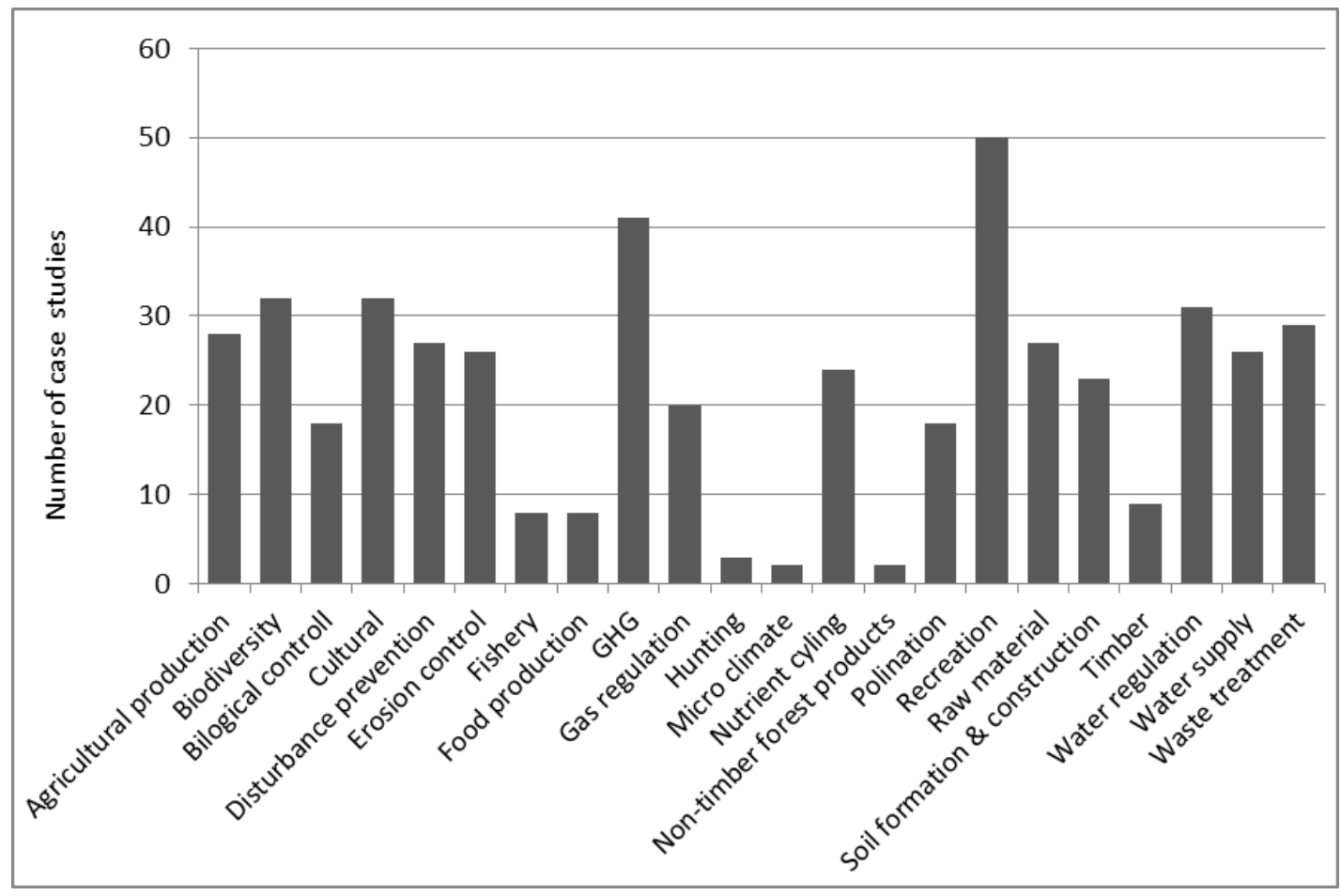

Figure 8: Frequency with which each ESS is mapped. 


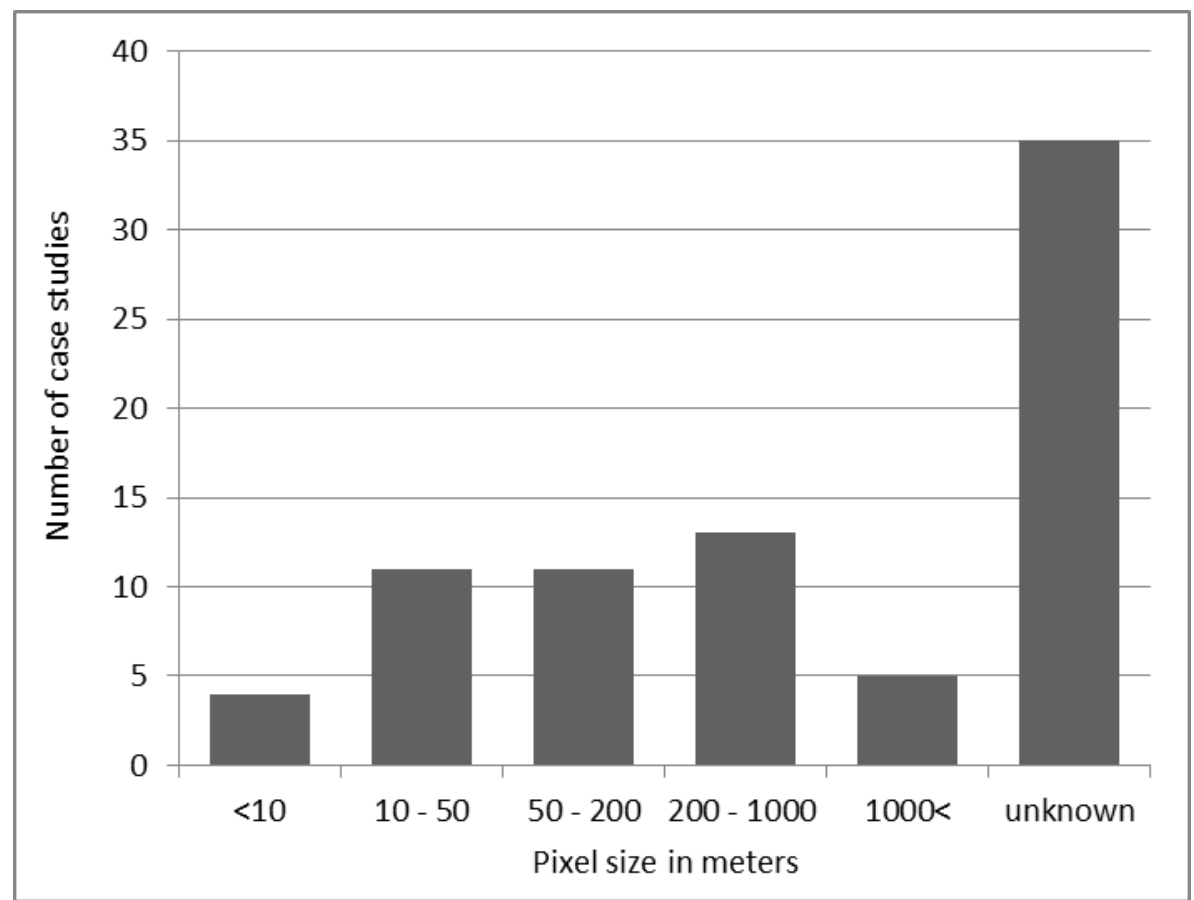

Figure 9: Resolution of ESS value maps.

\subsection{Mapping of Ecosystem Service Supply}

Methodologies used for mapping ESS supply can be divided into five main categories: (1) Onedimensional proxies for ESS, such as LCLU, (2) non-validated models: ecological production functions (or models) based on likely causal combinations of explanatory variables, which are grounded on researcher or expert assumptions, (3) validated models: ecological production functions, which are calibrated based on primary or secondary data on ESS supply, (4) representative data of the study area: data on ESS supply that is collected for the specific study area, and (5) implicit modelling of ESS supply within a monetary value transfer function: the quantity of ESS supply is modelled within the valuation of the ESS. Figure 10 shows the share of studies using each of these methodologies for assessing ESS supply.

(1) Most common are ESS maps that are based on one easily available proxy. Such ESS maps use one biophysical variable to map variations of ESS supply across space, mainly LCLU data, but also others such as water depth or slope angle are used. Approximately $52 \%$ of all studies map ESS based on proxies.

ESS models (also called ecological production functions) have also been widely used for mapping ESS. Such models assess the supply of ESS based on a set of spatial explanatory variables.

(2) In the absence of any primary data on ESS supply for model calibration and validation, researchers tend to build non-validated models for mapping ESS supply (23\% of all studies). These models are based on likely causal combinations of explanatory variables, but the causal combinations are grounded on researchers' or experts' assumptions or on information taken from the literature. No real world observations on ESS supply are used to calibrate the model or to test the model's validity.

(3) In contrast, validated models use primary or secondary data on ESS supply in order to calibrate the model parameters, for example by statistical regression analysis or by manual model optimisation. This approach is used by $34 \%$ of all studies. It is worth noting, however, that the distinctions between models that are calibrated based on primary or secondary data (validated models) and those that are based on researchers' assumptions (non-validated 
models) are not clear cut. Almost every complex ESS model relies to some extent on researchers' assumptions. Moreover, in the absence of data on ESS supply for the study area, some studies use data for calibration, which were obtained for a different spatial context and for different purposes.

(4) ESS maps that are based on representative data use a minimum of one real world observation to quantify ESS supply within each patch of the ESS supply map. The application of this approach is limited and has been used mainly either for small study areas or at coarse resolutions (Eigenbrod et al. 2010b). Approximately 13\% of all studies map at least one ESS based on representative data.

(5) A relatively small number of studies - typically with a strong environmental economics background - use implicit modelling to map ESS supply. Approximately $9 \%$ of the reviewed studies use this approach. Such studies use value functions that relate variation in unit ESS values to variation in the characteristics of the ecosystem, context and population of beneficiaries. Site-specific parameter values are plugged into the value function in order to derive a value estimate at every location of the study area. In applications in which the value function contains several biophysical variables that have a causal relationship with ESS supply, the model can be interpreted as providing an implicit modelling of ESS supply, although the ESS supply is not derived explicitly.

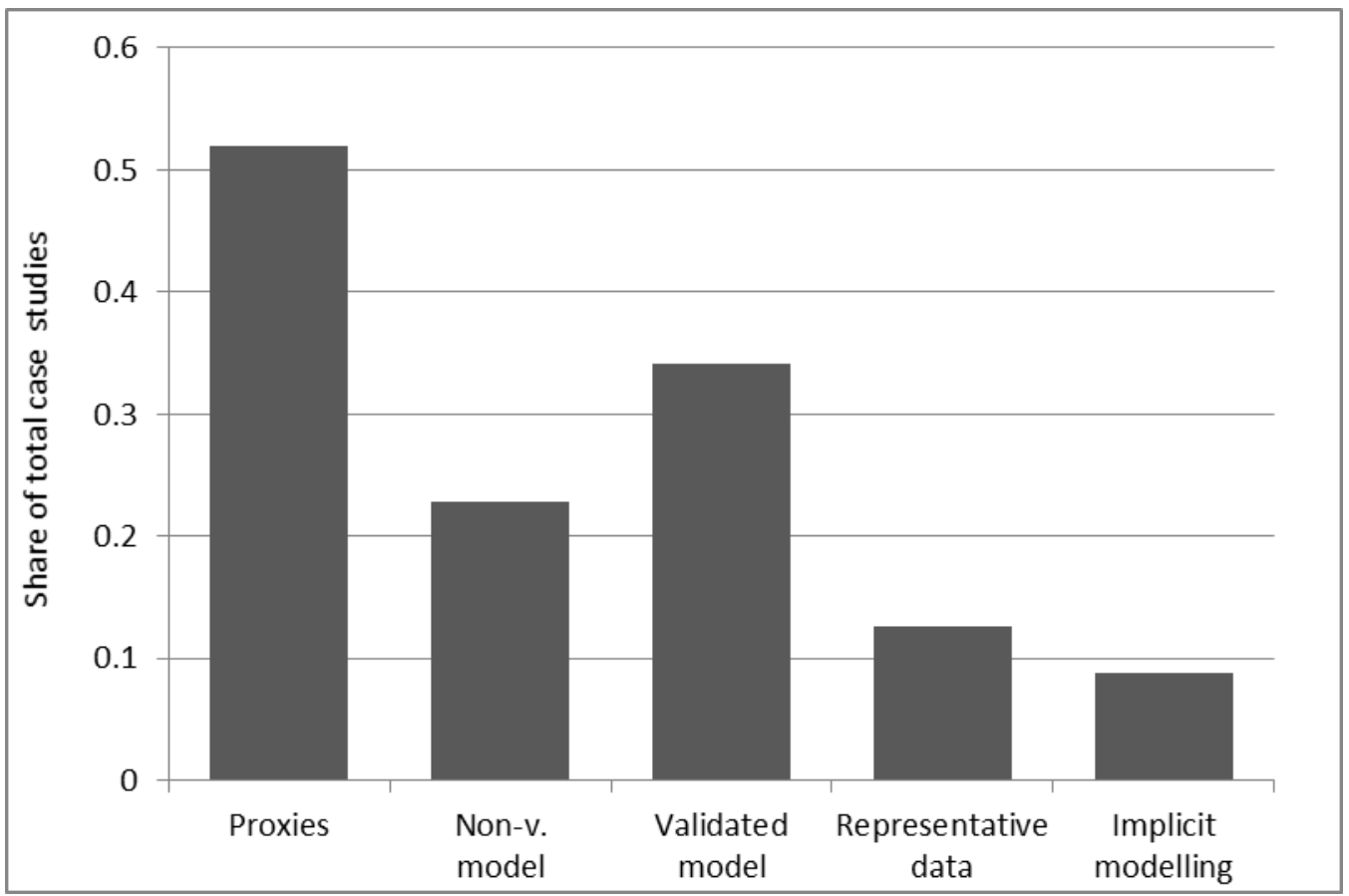

Figure 10: Share of studies using a specific methodology for mapping ESS supply.

\subsection{Mapping of Ecosystem Services' Values}

Mapping of ESS values requires that monetary values are assigned to mapped ESS provision. This can either be done by conducting a new primary valuation study for the case study area or by transferring values from existing studies for other similar study areas (known as value or benefit transfer). Primary valuation involves estimating the monetary value of the ESS supply of the case study area through the 
application of one or more market or non-market valuation methods. ${ }^{2}$ Value transfer involves transferring values from one or multiple study sites, for which the ESS has been valued, to the current study site (often termed policy site). Typically, the reason for performing value transfer is to obtain information on ESS values without conducting time consuming and costly primary valuation studies. In total $42 \%$ of the reviewed studies conduct primary valuation, whereas $84 \%$ use value transfer for at least one ESS. In order to map variation in ESS values, value estimates are then distributed across the study area using the methods described below.

In analogy to the value transfer literature, we distinguish between four different methodologies for distributing values across the study area: (1) unit values (2) adjusted unit values (3) value functions and (4) meta-analytic value function transfers. ${ }^{3}$

(1) In the unit value approach, a constant value per unit of ESS is applied across the study area. Thus, variations of ESS value across space result only from variations in ESS supply. Unit values are the predominant methodology for valuing ESS within the value mapping literature (78\% of all studies).

(2) The adjusted unit values approach adjusts values per unit of ESS across the study area using simple variables in order to account for spatial variations in value. Typically, such variables are population density, income levels or consumer price index. Thereby, such adjustments account for the number of beneficiaries of an ESS, the effect of income levels on willingness to pay, and differences in price levels. About $5 \%$ of all studies use adjusted unit values for ESS value mapping.

(3) Value functions are used to map values across the study area based on a function, which may contain multiple spatial variables. The value function is typically estimated within one primary valuation study, which may be conducted within or outside of the study area. It is then applied to the entire study area by plugging in site-specific parameter values into the value function. About $20 \%$ of all ESS value mapping studies use value functions.

(4) The meta-analytic value function transfer approach also transfers values to the entire study area by plugging in site-specific characteristics into a value function. In this case, however, the function is estimated through statistical regression analysis of the results of a number of primary valuation studies. Only $4 \%$ of the reviewed case studies use this methodology (see Figure 11).

2 Detailed information on the underlying theory and practical implementation of non-market valuation techniques can be found in a number of texts including Hanley and Spash (1993), Pearce et al. (1994) and Freeman (2003).

\footnotetext{
${ }^{3}$ For a general overview on the different value transfer methodologies see for example Navrud and Ready (2007)
} and Navrud and Bergland (2001). 


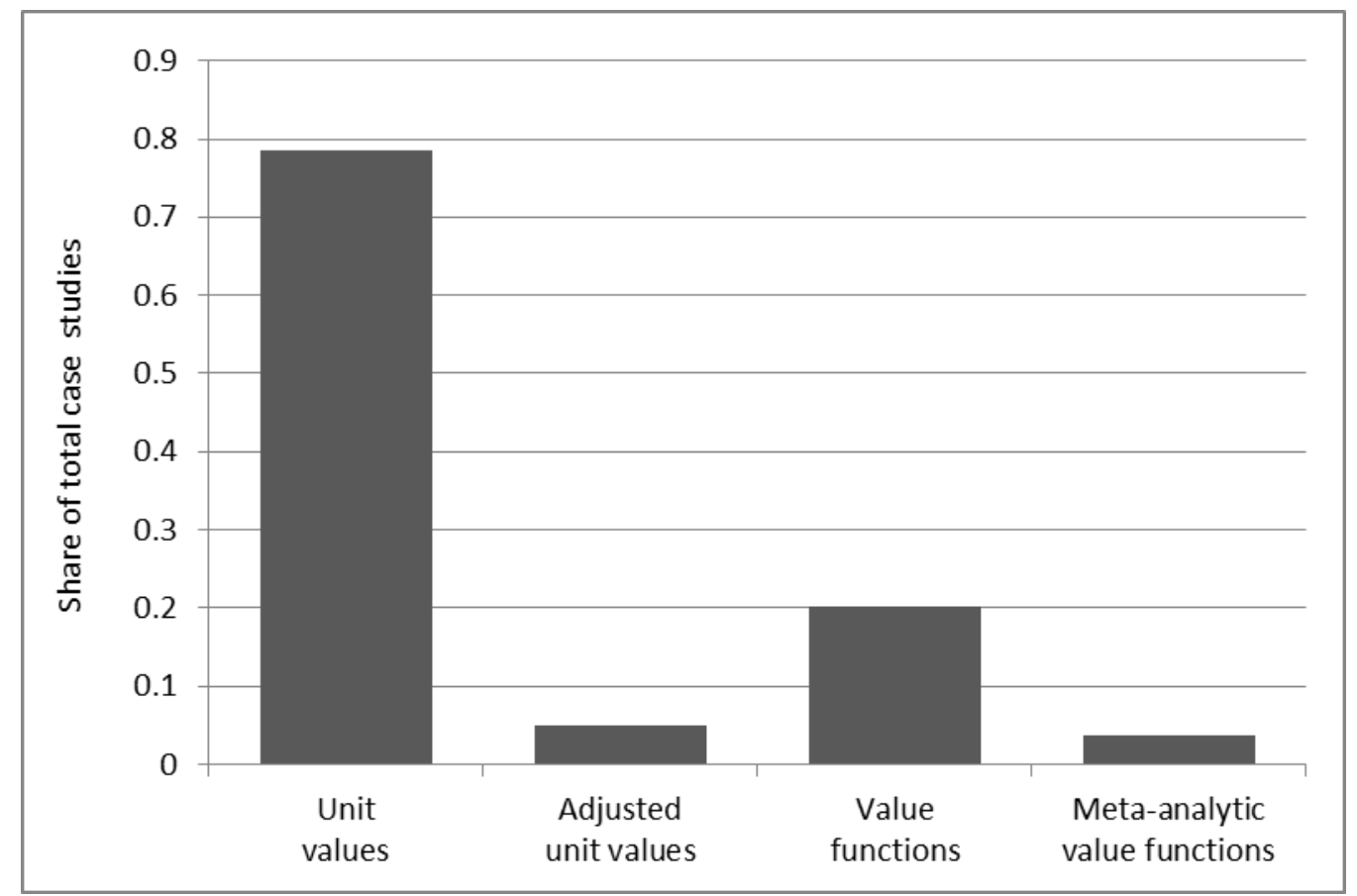

Figure 11: Share of studies using a specific methodology for valuing ESS.

\subsection{Combinations of Methodologies Applied in Literature}

By combining the two dimensions of ESS value mapping, we draw a methodology matrix and allocate all reviewed studies within this matrix according to the methodologies used for mapping ESS supply values (see Table 1$)^{4}$. In each cell of the matrix, we include abbreviations for the different ESS. Each abbreviation is followed by numbers, which refer to studies that map the specific ESS using the combination of methodologies indicated for that cell. The abbreviations and studies are listed in the lower part of the Table. Readers that are interested in a particular methodology or a particular ESS can find the references of the relevant studies listed in the Table.

\footnotetext{
${ }^{4}$ The classification of some studies was difficult (mainly the differentiation between validated and non-validated models) in cases for which not all relevant information is available in the published article. In such cases, we searched for further information within the mentioned references.
} 
Table 1: Matrix of methodologies used in literature for mapping ecosystem service values.

\begin{tabular}{|c|c|c|c|c|}
\hline Methodology & Unit Values & $\begin{array}{l}\text { Adjusted unit } \\
\text { values }\end{array}$ & Value functions & $\begin{array}{l}\text { Meta-analytic } \\
\text { value functions }\end{array}$ \\
\hline Proxies & 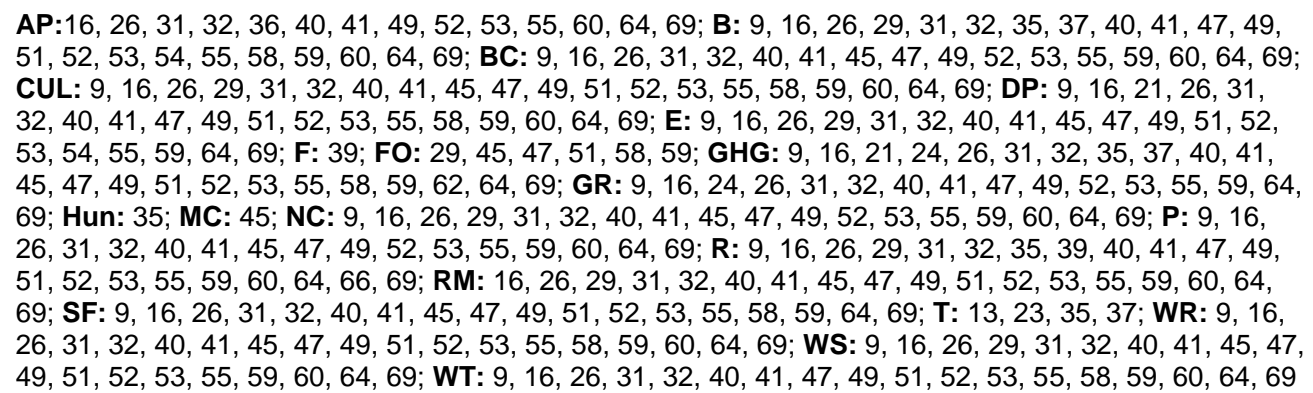 & $\begin{array}{l}\text { CUL: } 18 ; \text { Non-T: } \\
\text { 14; R: } 18 ; \text { T: } 14\end{array}$ & CUL: 11; R: 11, 24 & $\begin{array}{l}\text { CUL: 62; B: } 8 \text {, } \\
\text { 14, 62; F: 8; } \\
\text { Hun: 8; R: 14; } \\
\text { RM: 8; DP: 8, 62; } \\
\text { WT: 8, 62; WS: } \\
8,62\end{array}$ \\
\hline $\begin{array}{l}\text { Non-validated } \\
\text { models }\end{array}$ & 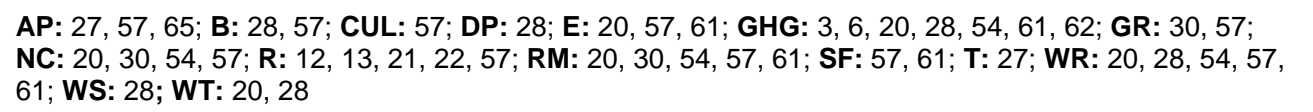 & CUL: 34 & AP: $63 ; \mathbf{R}: 2 ; \mathbf{T :} 12$ & \\
\hline $\begin{array}{l}\text { Validated } \\
\text { models }\end{array}$ & $\begin{array}{l}\text { AP: } 15,56 \text {; B: } 23 \text {; GHG: } 3,6,12,18,34,38,62 ; \text { GR: } 56 \text {; E: } 21,24 ; F: 1,39 ; \text { Hun: } 37 \text {; MC: } 34 \text {; NC: } 56 \text {; } \\
\text { R: 5, 12, 10; WR: } 24,25,33,34 \text {; WT: } 18,24,48\end{array}$ & WT: 34 & $\begin{array}{l}\text { AP: } 4,38,42 ; \text { DP: } 23 \\
\text { R: } 2,7,35,50 ; \text { T: } 38,42\end{array}$ & R: 62 \\
\hline $\begin{array}{l}\text { Representative } \\
\text { data }\end{array}$ & AP: 13, 18, 19; B: 21; GHG: 14; F: 39, 46; Non-T: 21; R: 39, 46; RM: 22; WS: 22 & R: 44 & AP: 35 & \\
\hline $\begin{array}{l}\text { Implicit } \\
\text { modelling }\end{array}$ & & & $\begin{array}{l}\text { AP: } 62 ; \text { CUL: } 23,43 \\
\text { 62; R: } 43,62 ; \text { DP: } 17\end{array}$ & CUL: $8 ; \mathbf{R}: 8,67$ \\
\hline \multicolumn{5}{|c|}{$\begin{array}{l}\text { AP: Agricultural production, B: Biodiversity, BC: Biological Control, CUL: Cultural (including Amenity), DP: Disturbance Prevention (including storm protection, flood protection and avalanche } \\
\text { protection), E: Erosion Control, F: Fisheries, FO: Food Production, GHG: Green House Gasses Regulation, GR: Gas Regulation (atmospheric chemical composition), Hun: Hunting, MC: Micro } \\
\text { Climate Regulation, NC: Nutrient Cycling, Non-T: Non-Timber Forest Products, P: Pollination, R: Recreation, RM: Raw Material, SF: Soil Formation, T: Timber, WR: Water Regulation, WS: Water } \\
\text { Supply, WT: Waste Treatment (including soil, air and water quality) }\end{array}$} \\
\hline \multicolumn{5}{|c|}{$\begin{array}{l}\text { 1. (Armstrong et al. 2003), 2. (Baerenklau et al. 2010), 3. (Bateman and Lovett 2000), 4. (Bateman et al. 1999), 5. (Bateman et al. 1995), 6. (Brainard et al. 2009), 7. (Brainard 1999), 8. (Brander et } \\
\text { al. 2011), 9. (Brenner et al. 2010), 10. (Bateman, Lovett, et al. 1999), 11. (Campbell et al. 2009), 12. (Chan et al. 2011), 13. (Chen et al. 2009), 14. (Chiabai et al. 2011), 15. (Coiner et al. 2001), 16. } \\
\text { (Costanza et al. 1997), 17. (Costanza et al. 2008), 18. (Crossman et al. 2010), 19. (Crossman and Bryan 2009), 20. (De-yong et al. 2005), 21. (Eade and Moran 1996), 22. (O'Farrell et al. 2011), } \\
\text { 23. (Grêt-Regamey et al. 2008), 24. (Guo et al. 2001), 25. (Guo et al. 2000), 26. (Helian et al. 2011), 27. (Holzkämper and Seppelt 2007), 28. (Ingraham and Foster 2008), 29. (Isely et al. 2010), 30. } \\
\text { (Jin et al. 2009), 31. (Konarska et al. 2002), 32. (Kreuter et al. 2001), 33. (Mashayekhi et al. 2010), 34. (McPherson et al. 2011), 35. (Moons et al. 2008), 36. (Naidoo and Adamowicz 2006), 37. } \\
\text { (Naidoo and Ricketts 2006), 38. (Nelson et al. 2009), 39. (O'Higgins et al. 2010), 40. (Petrosillo et al. 2009), 41. (Petrosillo et al. 2010), 42. (Polasky et al. 2008), 43. (Powe et al. 1997), 44. (Rees et } \\
\text { al. 2010), 45. (Sandhu et al. 2008), 46. (Scheurle et al. 2010), 47. (Seidl and Moraes 2000), 48. (Simonit and Perrings 2011), 49. (Sutton and Costanza 2002), 50. (Termansen et al. 2008), 51. (Troy } \\
\text { and Wilson 2006), 52. (Williams et al. 2003), 53. (Yoshida et al. 2010), 54. (Yu et al. 2005), 55. (Yuan et al. 2006), 56. (J. Zhang et al. 2011), 57. (M. Zhang et al. 201), 58. (W. Zhang et al. 2007), } \\
\text { 59. (Zhao et al. 2004), 60. (Zhao et al. 2005), 61. (Zhiyuan et al. 2003), 62. (Bateman et al. 2011), 63. (Naidoo and Adamowicz 2005), 64. (Viglizzo and Frank 2006), 65. (Anderson et al. 2009)66. } \\
\text { (Ghermandi et al. 2010), 67. (Ghermandi et al. 2011), 68. (Wei et al. 2007), 69. (Liu et al. 2010) }\end{array}$} \\
\hline
\end{tabular}


Almost half of the reviewed studies combine LCLU proxies with unit values (46\%). ${ }^{5}$ With reference to the well-known publication of Costanza et al. (1997), this is also referred as to the "Costanza Approach". Within this study, global ESS values are mapped by attributing mean values of multiple ESS per LCLU class from a number of primary valuation studies to a global LCLU data set. The only biophysical variable used to describe differences in ESS supply across space is LCLU (proxy). The ESS values per unit of ESS do not differ across space (unit value). This approach has been replicated multiple times at local to global scales and by using different valuation and LCLU data sets (Sutton and Costanza 2002; Troy and Wilson 2006). Besides that, several studies use LCLU in combination with unit values in order to complement their findings on a specific ESS, which they investigate more in depth. Typically, such studies focus on one or a small number of ESS using more detailed methods. Additional ESS values are then included by the rather simple combination of LCLU and unit values in order to provide a more comprehensive assessment of ESS values.

Validated models in combination with unit values are used by about $25 \%$ of all studies. For example, Guo et al. (2001) value forest water flow regulation by its positive effect on electricity production in a downstream hydropower plant. The total value estimate is distributed across the study area in accordance with the contribution to water flow regulation of each location in the study area. Thus, the value per unit of water retention does not differ across space (unit value). However, water flow regulation differs across space based on a model using vegetation, soil and slope angle as spatial explanatory variables. The model is calibrated based on "in-situ surveys and field experiments" (validated model). Brainard et al. (2009) model carbon sequestration in Welsh forests for live wood, wood products and soils. Carbon sequestration differs spatially due to variation in tree species and yield classes which are modelled based on several spatial variables such as climate data, soil types and legal status. The model is calibrated based on multiple forest records (validated model). Carbon is valued using one uniform value per ton sequestered carbon (unit value). Simonit and Perrings (2011) model the impact of wetlands on the water quality in Lake Victoria. Data for model calibration is not taken from the study area itself, but from "closely allied systems" (validated model). A uniform value is estimated per unit of nutrient retention based on an estimated impact on fish catch in the downstream lake (unit value).

The combinations of non-validated models with unit values (19\%) and representative data with unit values (10\%) are also used relatively frequently. Eade and Moran (1996) map recreational values based on the assumptions that the recreation service is distributed across the study area based on "distance and visibility from tourist areas". However, no reference is given on whether this relationship is based on any real world observation (non-validated model). The total recreational value estimate for the entire study area is then distributed in accordance to the mapped ESS distribution (unit value). Crossman et al. (2010) map agricultural production values based on yield statistics for the study area (representative data). An ESS value is attached to the yield by combining it with constant farmer net returns for each LCLU type (unit value). O'Higgins et al. (2010) map values for recreational clamming in a 1800 ha bay in Oregon, USA. Recreational use is quantified in a spatially explicit manner based on a comprehensive survey of the study area (representative data). A constant willingness to pay (WTP) value is attributed to each recreational user (unit value).

Besides unit values, value functions are the only valuation method used relatively often, mainly in combination with validated models (10\%). Polasky et al. (2008) model yields and net revenues of agricultural and timber products in Willamette Basin in Oregon, USA. The models on agricultural yields and timber production use land use, climate and soil data as explanatory variables. The models are

\footnotetext{
${ }^{5}$ Note that a number of studies use different methodologies for mapping values of different ESS.
} 
calibrated based on yield data (validated model). The net revenues of each land use are modelled spatially explicitly using a function that includes spatial variables, such as parcel location, slope and land use (value function). A number of studies use validated models to map recreational use, which is then valued based on travel cost models (Moons et al. 2008; Termansen et al. 2008; Bateman et al. 1999a; b). The recreational demand models use visitor survey data for model calibration. The value per visit (VV) is then modelled using a travel cost function, which results in different values per visit for different locations in the map (value function). Grêt-Regamey et al. (2008) model the impact of forest cover on avalanche protection based on avalanche probability, slope and land cover data. The model is calibrated based on avalanche records (validated model). Values are a function of avalanche risk reduction and property and human lives at risk (value function).

Other methodology combinations show relatively few applications. Implicit modelling of ESS supply within value functions is used by $6 \%$ of all studies. Costanza et al. (2008) map wetland values for storm protection. The value function for modelling marginal wetland values includes biophysical variables of storm probability, wind speed, storm swath and wetland area. Thus, the ESS storm protection is not explicitly modelled within an ESS model but still the ESS supply is quantified implicitly based on the biophysical variables within the value function. Powe et al. (1997) use a value function based on a hedonic pricing model for mapping recreational and amenity values of forests. The model, however, does also include forest characteristics in form of an access index, which correlate with recreational use. Thus, the value function quantifies the ESS implicitly.

About $4 \%$ of the reviewed studies combine value functions with non-validated models. For example, Baerenklau et al. (2010) map recreational values within a protected forest assuming that recreational use within the forest distributes equally from the access points and that landscape value is dependent on its visibility. However, this relationship is based on the researchers' assumptions and not on real world observations (non-validated model). Values are a function of visitor numbers, visibility and travel costs estimated for each access point (value function).

Meta-analytic value functions are still relatively rarely used within ESS value mapping, although they have gained increasing attention within traditional individual site specific value transfer. About $4 \%$ of all studies use meta-analytic value functions in combination with proxies and about $3 \%$ conduct implicit modelling within the meta-analytic value function. For example, Bateman et al. (2011) mapped multiple wetland ESS values based on a meta-analytic value function. The only biophysical variable causing values to differ spatially is the distinction between inland and coastal wetlands, which we classify in our matrix as a proxy. The meta-analytic value function used by Ghermandi et al. (2011) to map global coastal recreational values includes multiple biophysical variables that correlate with recreational use (e.g. climate, biodiversity and accessibility). Therefore, an implicit modelling of the ESS recreation within the meta-analytic value function is conducted.

Proxies in combination with value functions are used by $3 \%$ of all studies. Guo et al. (2001) mapped recreational values by using a travel cost model for valuation (value function). However, the only biophysical feature affecting spatial value distribution is LCLU (proxy).

Only one study uses non-validated models in combination with adjusted unit value transfer. McPherson et al. (2011) mapped amenity values of urban trees by assuming that amenity depends on tree size. However, no primary or secondary data is used for calibration or validation of this relationship (non-validated model). A value per large tree is taken from one hedonic pricing study. The value per tree is then adjusted by one variable, the number of beneficiaries in terms of residential housing density. Thus, we classified this approach as an adjusted unit value. 
We identified some correlations between the methodology used and other study characteristics. However, due to the limited number of studies for some methodological combinations, it is difficult to conclude an overall trend. Typically, studies that use a combination of proxies and unit values map values of multiple ESS (mean 10), whereas more complex methodologies result in fewer ESS being addressed (an average 1-2 ESS per study). ${ }^{6}$ Studies that attempt to cover all ESS values are commonly mapped using the combination of proxies and unit values.

We can only identify a few concentrations of certain combinations of methods being used for mapping values of a specific ESS. Recreational values are relatively frequently mapped by a variety of different methodology combinations other than proxies and unit values. Some studies use validated models (8), especially in combination with unit values (3) or value functions (4). Some applications use nonvalidated models in combination with unit values (5) and also implicit modelling within (meta-analytic) value functions (4). Some case studies map waste treatment by validated (3) or non-validated models (2), both in combination with unit values. Water regulation (4/5) and GHG (7/7) are mapped frequently by validated or non-validated models, always in combination with unit values. Also erosion is mapped by non-validated (3) and validated models (2) in combination with unit values. For raw materials we found five case studies using non-validated models in combination with unit values. Agriculture has some applications of non-validated (4) and representative data (4); mainly in combination with unit values but also some applications of validated models exist (5), mainly in combination with value functions.

There are also some patterns with respect to the policy application that is addressed by a study and the methodology used. Green accounting is dominantly mentioned within studies using unit values, either in combination with proxies, non-validated models or representative data. Resource allocation and land use policy evaluation are mentioned frequently within studies using unit values or value functions.

The spatial extent of the study area tends to be smaller for studies using value functions and for studies using validated or non-validated models. The largest mean study areas are found for studies using proxies. Finally, we identified a temporal trend towards the application of more sophisticated methodologies. Only $47 \%$ of all studies published after 2007 use proxies or non-validated models combined with unit values or adjusted unit values. For the sum of all other combinations of methods, this share amounts to $75 \%$.

\subsection{Accuracy and Precision in ESS Values Mapping}

An important and insufficiently assessed issue in mapping ESS values is the accuracy and precision of such maps. If ESS value maps are used to support policy decisions, policy-makers need to know how reliable the mapped values are. How close are the estimated values to the real ESS values? Does the value map provide accurate and precise site-specific value estimates, or does it display coarse trends at the landscape level, or does it only give a rough estimate of total ESS values in the case study area?

Reviewing the literature, we found that about one third of studies do not address the question of accuracy and precision of their mapped values at all. About $58 \%$ of all studies at least discuss potential value mapping errors qualitatively. However, only a minor share of the reviewed studies give quantitative information on error margins of their results either by displaying parameter estimates from the statistical analysis, by estimating boundaries within which the actual values may most likely

\footnotetext{
${ }^{6}$ Only meta-analytic value functions in combination with proxies show a higher mean number of about five ESS mapped per case study. However, only three case studies were found for this combination of methods.
} 
lie, by conducting sensitivity analyses or by comparing predictions with real world observations (see Figure 12). Due to the limited number of studies quantifying error margins, it is not possible to draw conclusions on which method may deliver the most accurate and precise value maps. However, some conclusions can be drawn from the value transfer and ESS modelling literature.

Errors in ESS value mapping may result from inaccurate/ imprecise mapping of ESS supply and their values. Both of them can be subdivided into four sources of errors: (1) errors in the primary ESS supply and value estimates, (2) uniformity, generalisation or interpolation errors, (3) sampling or publication errors and (4) regionalisation or extrapolation errors (Eigenbrod et al. 2010a; b).

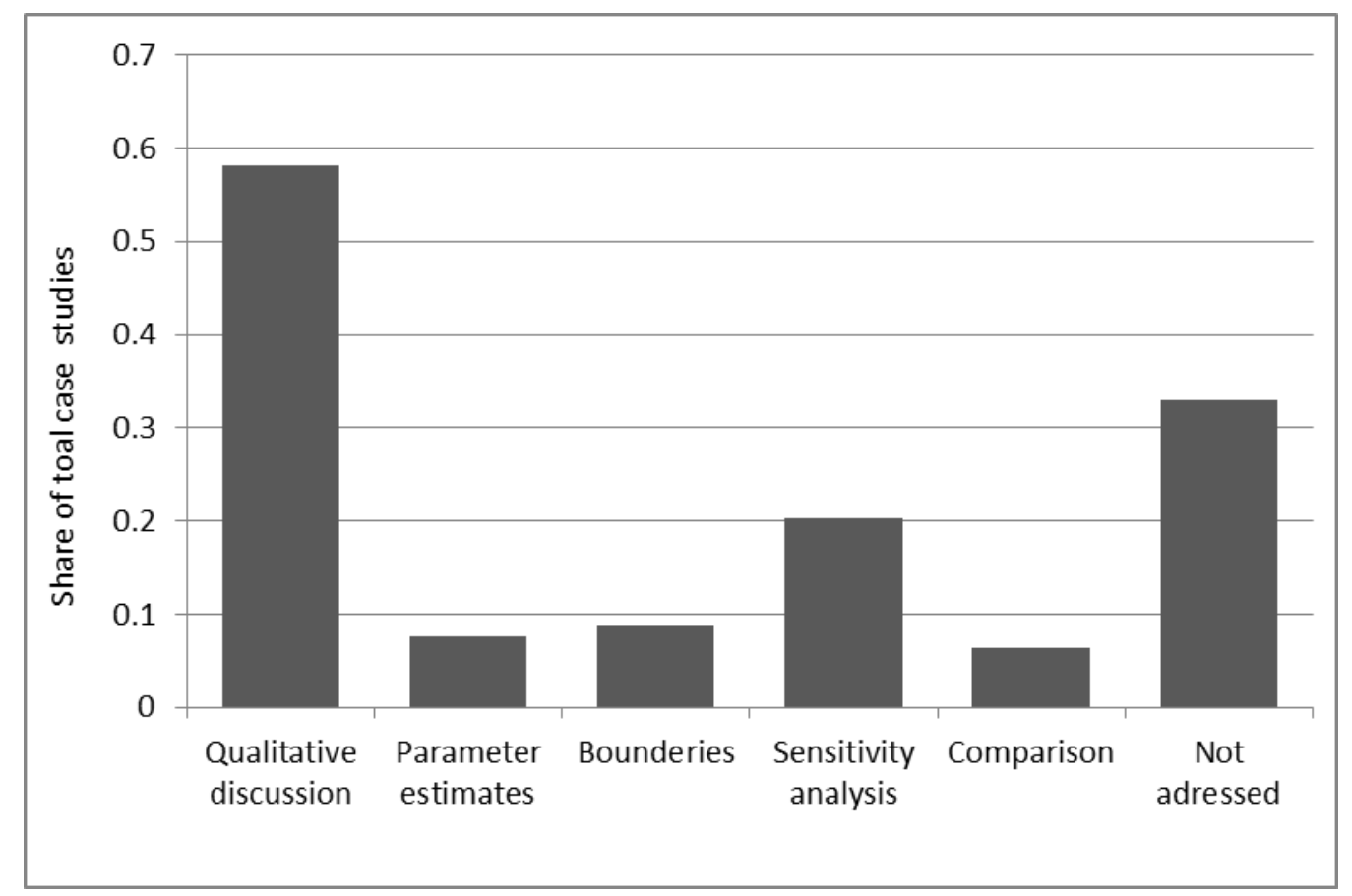

Figure 12: Assessment of results accuracy.

(1) Errors in primary data collection may depend on the methods and care in taking samples. Meta-analyses report that sample results can be statistically significantly different for different primary data collection techniques, both for ESS measurements and primary valuation.

(2) Uniformity, generalisation or interpolation errors result from the fact that ESS supply and its values are considered to be constant across heterogenic ecosystems, even though ESS supply and its values vary due to multiple factors that are not observable or are not accounted for in the mapping exercise.

(3) Sampling or publication bias errors result from the fact that primary data may not be representative for the study area. Reasons for this include the higher publication rates of statistically significant and prior expectation supporting results and non-representative study site selection due to researchers' interests and research funding policy (Stanley and Rosenberger 2009; Rosenberger and Johnston 2009).

(4) Regionalisation or extrapolation errors may occur when values are transferred between different areas that are characterised by different ESS supply and demand. Due to limited data availability, primary data may often be taken from samples outside of the study site and therefore, their transferability may be limited (Eigenbrod et al. 2010a; b; Rosenberger and Phipps 2007; Johnston and Rosenberger 2010).

The few studies quantifying accuracy of their mapped values show considerable errors. Konarska et al. (2002) use LCLU proxies and unit values to compare how different resolutions of LCLU data sets 
influence the results of total ESS values in the US. The total value estimate increased by a factor of two for the finer resolution, because the share of high value and highly fragmented LCLU increased. Using a meta-analytic value function for mapping wetland values across the EU, Brander et al. (2011) report $95 \%$ confidence intervals of the total wetland value predictions per country. The lower bound differs to the upper bound up to a factor of two. Costanza et al. (1997) conduct sensitivity analysis on the ESS value estimates that they attribute to the different biomes in order to map global ESS values. The total value estimate differs by a factor of more than three. Costanza et al. (2008) conducted a sensitivity analysis by setting a maximum marginal value for a wetland. As a result, the total value estimate differs by a factor of almost seven. By applying different valuation methodologies for mapping water supply values, O'Farrell et al. (2011) estimate that total values differ by a factor of about six.

The reported error margins here are the sum of mean errors over large areas and give no information on the precision and accuracy for any site specific estimate. Such errors may be far higher. Eigenbrod et al. (2010a; b) estimate errors associated with ESS mapping using land cover proxies. They make a comparison between ESS maps that assume a constant ESS supply per LULC class and maps that are based on real world observations of ESS supply. The correlations (Spearman's rho) between the predicted and observed provision of ESS are low ( 0.37 for biodiversity, 0.42 for recreation and 0.57 for carbon storage). Combining their results with unit values in order to derive an ESS value map would result in even higher errors, as values per unit of ESS supply may again differ across space. However, they find that including additional explanatory variables for population and accessibility increased the correlation between predicted and observed data for recreation to at least 0.50 . Brookshire et al. (2007) assess the impact of uncertainties in economic valuation and biophysical models on the value of water resources in a river basin for agricultural, domestic and conservation use. They conclude that uncertainties result from the valuation and population predictions rather than from the biophysical ESS modelling. 
Table 2: Evaluation of methodologies.

\begin{tabular}{|c|c|c|c|c|}
\hline Methodology & Unit values & Adjusted unit values & Value functions & Meta-analytic value functions \\
\hline Proxies & $\begin{array}{c}\text { Simple / } \\
\text { Low data requirements / } \\
\text { Low precision/ } \\
\text { Intransparent quality }\end{array}$ & $\begin{array}{c}\text { Simple / } \\
\text { Low data requirements / } \\
\text { Low precision/ } \\
\text { Intransparent quality }\end{array}$ & $\begin{array}{l}\text { Medium complexity / } \\
\text { Medium data requirements / } \\
\text { Medium precision/ } \\
\text { Intransparent quality }\end{array}$ & $\begin{array}{l}\text { Medium complexity / } \\
\text { Medium data requirements / } \\
\text { Medium precision/ } \\
\text { Transparent quality }\end{array}$ \\
\hline $\begin{array}{l}\text { Non-validated } \\
\text { models }\end{array}$ & $\begin{array}{l}\text { Medium complexity / } \\
\text { Medium data requirements / } \\
\text { Medium precision/ } \\
\text { Intransparent quality }\end{array}$ & $\begin{array}{l}\text { Medium complexity / } \\
\text { Medium data requirements / } \\
\text { Medium precision/ } \\
\text { Intransparent quality }\end{array}$ & $\begin{array}{c}\text { High complexity / } \\
\text { Medium data requirements / } \\
\text { High precision/ } \\
\text { Intransparent quality }\end{array}$ & $\begin{array}{l}\text { High complexity / } \\
\text { High data requirements / } \\
\text { High precision/ } \\
\text { Transparent quality }\end{array}$ \\
\hline $\begin{array}{l}\text { Validated } \\
\text { models }\end{array}$ & $\begin{array}{c}\text { Medium complexity / } \\
\text { Medium data requirements / } \\
\text { Medium spatial explicitness, } \\
\text { Transparent quality }\end{array}$ & $\begin{array}{c}\text { Medium complexity / } \\
\text { Medium data requirements } \\
\text { Medium spatial explicitness, } \\
\text { Transparent quality }\end{array}$ & $\begin{array}{l}\text { High complexity } \\
\text { High data requirements } \\
\text { High spatial explicitness } \\
\text { Transparent quality }\end{array}$ & $\begin{array}{c}\text { High complexity } \\
\text { Very high data requirements } \\
\text { High spatial explicitness } \\
\text { Very transparent quality }\end{array}$ \\
\hline $\begin{array}{l}\text { Representative } \\
\text { data }\end{array}$ & $\begin{array}{c}\text { Simple / } \\
\text { High data requirements } \\
\text { Medium spatial explicitness } \\
\text { Intransparent quality }\end{array}$ & $\begin{array}{c}\text { Simple / } \\
\text { High data requirements } \\
\text { Medium spatial explicitness } \\
\text { Intransparent quality }\end{array}$ & $\begin{array}{l}\text { Medium complexity / } \\
\text { High data requirements } \\
\text { High spatial explicitness } \\
\text { Intransparent quality }\end{array}$ & $\begin{array}{l}\text { Medium complexity / } \\
\text { Very high data requirements } \\
\text { High spatial explicitness } \\
\text { Intransparent quality }\end{array}$ \\
\hline $\begin{array}{l}\text { Implicit } \\
\text { modelling }\end{array}$ & - & - & $\begin{array}{c}\text { Medium complexity / } \\
\text { Medium data requirements } \\
\text { Medium spatial explicitness } \\
\text { Intransparent quality }\end{array}$ & $\begin{array}{c}\text { Medium complexity } \\
\text { High data requirements } \\
\text { Medium spatial explicitness } \\
\text { Transparent quality }\end{array}$ \\
\hline
\end{tabular}


For conventional value transfer most studies find site specific transfer errors between 0 and $100 \%$ (Eigenbrod et al. 2010b), but also higher errors are reported. Some authors argue that function transfers may result in lower transfer errors, even though evidence is mixed (Akter and Grafton 2010). In general, transfer errors tend to increase if study sites and policy sites are more heterogenic. However, due to the potential of (meta-analytic) value function approaches to make adjustments that reflect site-specific characteristics, these methods tend to be superior to (adjusted) unit values transfer in cases where sites differ heavily (Eigenbrod et al. 2010b). Some studies compare meta-analytic value function transfer with value function transfer, but do not reach a consensus on which method is preferable. The accuracy of (meta-analytic) value function transfer depends on the quality of the primary research being used to calibrate the value function and the available explanatory variables (Johnston and Rosenberger 2010). If meta-analytic value functions are only based on few observations and explanatory variables, they are likely to produce inaccurate predictions. A potential source of transfer error is that most (meta-analytic) value functions do not (or insufficiently) include site-specific bio-physical indicators in order to account for differences in ESS supply (Rosenberger and Phipps 2007; Johnston and Rosenberger 2010).

\subsection{Discussion of Methodologies}

Currently no consensus exists in the literature on which ESS mapping method is best to use for a specific purpose and under specific circumstances. Several factors may determine the choice of methodology, such as data availability, the ESS assessed, study area characteristics, the available resources, the policy context and the scientific purpose of the study. Advantages and disadvantages of each combination of methods depend heavily on the quality and the background of the individual study. Nevertheless, we evaluate each methodology combination by giving a tentative quality judgement on their advantages and disadvantages (see Table 2).

The different policy applications of ESS value mapping may demand different requirements in terms of accuracy and precision. If results are used for green accounting, an accurate overall value estimate of the entire study area's ESS may be desired. However, precision - meaning the accuracy of value estimates for each pixel of the map - may be of minor importance. Also land use policy evaluation may require accurate total value estimates of the different land use scenarios rather than precise value maps. In contrast, if results are used for resource allocation or for designing spatially explicit payments schemes for ESS accuracy but also precision are of greater importance. In any case, if results are used for real policy support, comprehensiveness in terms of ESS assessed is of major importance. If relevant ESS are not covered within the value map, it may alter the ranking order of alternative policy options (De Groot et al. 2010).

The advantage of LCLU proxies and unit values is that such data is easy to obtain. However, their correlation with location specific ESS supply and ESS values may be limited (Eigenbrod 2010a; b). The assumptions of uniform ESS supply and values across the same land covers, as used by Costanza et al. (1997) and repeated by many others, can be considered as a huge simplification (Plummer 2009; Eigenbrod et al. 2010a). It may hold for small and homogeneous case study areas and for some ESS, which by their nature are less prone to spatial variations in their supply and values. For example, it could be considered that spatial variations are low for agricultural yields and their values, if the study area is characterised by relatively similar climate and soil properties. In contrast, recreational use may even differ strongly across a relatively small homogenous forest due to limited diffusion of visitors away from access points. Nevertheless, LCLU proxies and unit values may still result in an accurate overall value estimate of entire study area's ESS, if correct mean values per LCLU are applied. Thus, it may be appropriate for green accounting and land use policy evaluation at a broad scale, but offers 
little information for a specific location on the map. Nevertheless, if mean values are transferred that were derived within totally different spatial contexts without adjusting them to the case study area's characteristics, the information provided may be low; both in terms of precision and accuracy (Plummer 2009; Tallis and Polasky 2009).

Validated and non-validated models have the advantage that they allow the mapping of ESS supply more precisely across larger and heterogeneous areas by accounting for a number of spatial variables. For example, adjusting water retention services spatially based on slope, soil and LCLU (Guo et al. 2001) may allow for more precise ESS maps than if only mean retention capacities per LCLU class are considered. However, the application of ESS models may be limited due to the complexity and effort in model construction and due to the unavailability of consistent comprehensive ESS indicators, especially for larger study areas. Applied models differ strongly in their complexity and the extent to which they incorporate site-specific characteristics. This may result in a wide range of accuracy and precision. Mapping ESS based on non-validated models can be considered as a pragmatic approach that combines the best available knowledge (De Groot et al. 2010). However, the quality of nonvalidated models remains unknown and depends heavily on the researchers' judgment regarding the causal relationships between variables. In contrast, validated models allow for validity testing by comparing the model predictions with real world observations. The share of studies that do not discuss the issue of mapping errors is especially high for studies using non-validated models (almost 60\%), in particular in combination with unit values. $67 \%$ of these studies do not give any reference to the potential errors of their ESS value map.

Representative data on ESS supply can result in very accurate and precise maps of ESS supply if samples are carefully collected. However, data collection is a very time consuming procedure. Therefore, its application is limited to small case study areas, coarse resolutions or to ESS for which such data is available in official statistics, such as timber and agricultural production.

Implicit modelling has the advantage that it allows research with a limited ecological background to include bio-physical indicators as explanatory variables in (meta-analytic) value functions. This approach can thereby account for variations in ESS supply and the value per ESS unit at the same time. This may result in more precise ESS value maps. For example, in a meta-analysis on forest values, Zandersen and Tol (2009) used not only strictly value- determining explanatory variables (such as GDP per capita and other socioeconomic characteristics) but also biophysical variables which correlate with ESS supply, such as fraction of open land, biodiversity and forest age diversity. However, modelling ESS supply and its value simultaneously introduces additional complexity, which may result in less accurate and spatially explicit ESS value maps, than if each would be estimated in separate models. The number of variables used within meta-analytic value functions is limited by the availability of primary value estimates used for the regression analysis. ESS that are not frequently valued, such as most regulating services, can therefore only be assessed by relatively simple meta-analytic value functions. Consequently, it may be of advantage to model ESS supply and values separately. If spatial variations in ESS supply are already explained, meta-analytic value functions may predict remaining spatial variations of values per unit of ESS supply more efficiently.

The specific strengths and weaknesses of different value transfer methodologies are discussed widely in value transfer literature (see for example Brouwer (2000), Navrud and Ready (2007), and Johnston and Rosenberger (2010)) and remain similar for ESS value mapping, but with some further specifications. The way in which these methods account for value-determining spatial characteristics is in particular relevance if values are mapped across large case study areas, as study areas tend to be more heterogeneous with size. Furthermore, accounting for spatial variations is in particular of 
importance for ESS value mapping, as one of its main purposes is to reveal how values differ across space. Whereas unit value applies one unique value per unit of ESS supply, adjusted unit values allow the adaptation of values across space by selected variables, such as income levels or the number of beneficiaries. The precision of an ESS value map produced using adjusted unit values may therefore be higher. For example, the value of flood control may not be constant across space but depends on property values at risk (De Kok and Grossmann 2010); or the amenity value of trees depends on the number of people benefiting from this service (McPherson et al. 2011).

ESS values may differ spatially due to further spatially variable characteristics such as the availability of substitutes and differences in human preferences across dissimilar sociocultural groups. (Metaanalytic) value functions allow such factors to be incorporated in the value mapping exercise and may thereby deliver more accurate and precise ESS value maps, especially for heterogenic study areas (Bateman and Jones 2003; Johnston and Rosenberger 2010; Rosenberger and Phipps 2007; Nelson and Daily 2010). However, this approach is generally more complex and time consuming to develop and requires comprehensive data sets of the explanatory variables across the entire study area, which may limit their application.

Typically, value functions are estimated for a specific location. However, parameters of the variables may be different in other locations, especially, if values are transferred across national or cultural borders (Johnston and Rosenberger 2010). This may limit the accuracy and precision of value functions for larger case study areas. For example, Moons et al. (2008) value forest recreation for a suburban region in Belgium using a travel cost model. The model is estimated based on local survey data, which may capture the local circumstances, but may be less accurate if applied within a very different spatial context.

An advantage of meta-analytic value functions is that they are based on multiple primary estimates, which can be collected across a large area and which use diverging valuation methodologies. Metaanalytic value functions are thereby able to capture the impacts of greater heterogeneity in site and context variables and methodologies in primary valuation studies (Bateman and Jones 2003; Brander et al. 2010). There is some evidence to suggest that meta-analytic value functions outperform other value transfer techniques, if sites differ strongly and if the number of primary valuation studies used for estimating the value function is large (Rosenberger and Phipps 2007). This suggests that metaanalytic value functions may be favourable for value mapping and that its potential may increase as the body of available primary valuation studies continues to grow. Furthermore, meta-analytic value functions allow the comparison of predictions with real world observations and thereby for quantification of prediction errors. However, meta-analyses require broad and quantitative databases of primary value estimates, which is a time consuming procedure and which may limit its application for ESS that are less widely valued.

\section{Future Prospects in ESS Value Mapping}

There are several issues within ESS value mapping that are of interest for future research. The challenge is to make ESS value maps more accurate, more precise and more comprehensive and to tailor them to support decision making. Finally, the role of biodiversity and ecosystems resilience in ESS provision remains insufficiently understood and has not been incorporated into ESS value maps.

The barriers to developing highly accurate ESS value maps are manifold. ESS values emanate from the spatial interaction of natural, human, social and built capital. Capturing these interactions is the principal challenge in ESS value mapping. Mapping of ESS and their values is dependent on 
quantitative, comprehensive and high resolution input data for all kinds of capital underlying the provision of ESS (social, human, built, and natural). Such data is required for both, as explanatory variables within ESS models and for model calibration. With improved remote sensing technologies and with continuous sampling, this data pool can be expected to grow in quantity, quality and spatial resolution. Efforts are required to harmonise available data and to construct online meta-databases to enhance access, such as the initiatives of the "The Ecosystem Services Partnership" (ESP) (http://www.es-partnership.org/esp) and "Earth Economics" (http://www.eartheconomics.org/). Quality and reporting standards for primary data collection have been repeatedly proposed in order to allow easier statistical assessments (Eigenbrod et al. 2010b; Rosenberger and Phipps 2007; Johnston and Rosenberger 2010). Furthermore, still little is known about many spatial determinants of ESS supply and its values. For example, how values differ across space due to differences in institutions and attitudes (Kotchen and Reiling 2000; Spash and Vant 2006; Pritchard et al. 2000), how different ESS are interlinked and how biodiversity contributes to ESS supply (Nicholson et al. 2009).

Accounting for the determinants of both ESS supply and its values requires a deeper integration of the disciplines involved (Bockstael et al. 2000). Still, many studies take rather mono-disciplinary approaches and only a limited number combines the strengths of multiple perspectives. Studies that are dominated by an ecological perspective tend to use sophisticated ESS models, but then apply rudimentary unit values methodologies. In absence of case specific valuation data, many studies use quickly derived value estimates, such as expenditure data, replacements costs and market prices for different ESS, but without any reference to the meaning and accuracy of such different value measures. On the other hand, studies that are dominated by an economic perspective may focus on the valuation process, but tend to rely on LCLU proxies or implicit modelling for ESS quantification. Within ESS modelling, attention needs also to be given to the definition and distinction of different ESS in order to avoid double counting and in order to fit model results into environmental economic valuation metrics.

Covering values of all relevant ESS is of great importance for policy decision support. Comprehensive ESS value maps allow the identification of trade-offs and synergies between different ESS values. Thereby, land use policies can be identified, that maximise total ecosystem service values (Tallis and Polasky 2009; De Groot et al. 2010). However, due to the complexity and the interdisciplinary nature of such research, there tends to be a trade-off between comprehensive inclusion of ESS and the accuracy and precision of the analysis. Typically, studies mapping values of multiple ESS combine simple LCLU proxies with unit values. It is not only a challenge to combine multiple models of ESS, but also to link them by creating meta-ESS models that include the feedbacks and linkages between different ESS. Difficulties are faced in harmonising input and output variables of different models (Tallis and Polasky 2009; Nicholson et al. 2009).

Furthermore, the policy orientation of many studies is still poor. Only about $35 \%$ of the reviewed studies evaluate some kind of scenario that may allow for policy evaluation. For giving guidance for policy makers, ESS value maps need to be linked to future policy assessments. Quantification and reporting of error margins in mapped values is still poor. If policy makers want to base their decisions on ESS value maps, they need to know about the uncertainties and error margins related to such maps. Therefore, validating mapped values against real world observations is indispensable (De Groot et al. 2010).

Finally, still little is known about the role of biodiversity and ecosystem resilience. The recent attempts of employing the concept of ESS for arguing in favour of biodiversity protection have only partly been successful. Evidence on correlations between biodiversity and ESS supply are mixed (Cardinale et al. 
2002; 2012; Maes et al. 2011b; 2012). However, the contribution of biodiversity to ecosystem resilience (its capacity to resist disturbances) and insurance values (the value of ensuring future ESS supply) are as yet hardly quantified. The often non-linear and multi-scale relations between measurable bio-physical quantities, ESS and biodiversity are not yet sufficiently understood. When and how drivers and pressures on ESS and biodiversity hit tipping points, beyond which ecosystems shift into a less desirable state is a critical question in ESS mapping and valuation. The rate of substitutability between different ESS and man-made capital, which is implied by their derived monetary values, changes drastically if thresholds are reached. Their incorporation into environmental valuation and policy scenario analysis is of critical concern for ensuring sustainable policy recommendations (De Groot et al. 2010; Nelson and Daily 2010).

ESS value mapping is gaining increased attention in current research and there are a number of initiatives progressing in ESS value mapping. The TEEB project (http://www.teebweb.org/) is mapping global ESS values based on LCLU proxies, but transferring values based on meta-analytic value function (TEEB 2010). Similarly, the AIRES project ${ }^{7}$ (http://www.ariesonline.org/) develops value up-scaling methodologies in order to derive more accurate ESS value maps. The UK NEA (National Ecosystem Assessment) (http://uknea.unep-wcmc.org/Home/tabid/38/Default.aspx) maps ESS values of agricultural and timber products, carbon storage and recreation across the UK. It combines different methodologies for mapping ESS supply, from comprehensive agricultural production data to validated production functions for timber, carbon storage and recreation (Bateman et al. 2010). The InVest tool (http://www.naturalcapi talproject.org/InVEST.html) aims at combining the capacities of researchers with different disciplinary backgrounds, in order to derive qualitative ESS supply and value maps for multiple ESS by combining different models and valuation methodologies (Tallis and Polasky 2009).

\section{Conclusion}

With the emergence of advanced GIS technology, spatial issues in environmental valuation have gained increasing attention and the importance of spatial relationships in ESS valuation has become widely recognised. The number of studies mapping ESS values by displaying how ESS values vary across space has grown exponentially in recent years. As compared to traditional site-specific valuation, ESS value mapping offers additional information by displaying trade-offs and synergies of alternative policy scenarios and enables the identification of preferable locations for policy measures.

Studies that map ESS values differ widely in terms of their spatial scope, purpose, disciplinary foundations and by the ESS assessed. A great variety exists in the methodologies used for revealing how ESS supply and values vary across space. Spatial variations in ESS values can be assessed by estimating spatial variations in ESS supply, the value per unit of ESS or through a combination of both of these determinants. In this paper, we developed a matrix for classifying studies with respect to the methodologies used for ESS value mapping. Methodologies for ESS supply mapping include onedimensional proxies, validated and non-validated models, representative data and implicit modelling within (meta-analytic) value functions. Methodologies for the spatial distribution of ESS values include unit value, adjusted unit value, value function and meta-analytic value function. However, until now, no consensus exists on which methodology is best to use for what purpose.

Accuracy and precision are issues of great concern in ESS value mapping, which is yet insufficiently addressed in literature. Only a minor proportion of the reviewed studies assess this issue in a

7 AIRES refers to ARtificial Intelligence for Ecosystem Services. For further information, consult http://www.ariesonline.org/. 
quantitative manner, even though evidence shows that error margins can be large. Due to coarse assessments and large uncertainty within mapped values, some studies may not reliably provide any site-specific policy suggestions. The "Costanza approach" of combining LCLU proxies with unit values, which were derived in specific contexts, may display coarse trends at landscape level, but may give only limited information for site-specific assessments. The Costanza et al. (1997) study represents a significant step in the mapping of ESS values, but its limitations have been widely discussed in literature and are also largely recognised within the study itself. The current challenge is to develop spatially explicit models of ESS supply combined with spatially explicit (meta-analytic) value functions; both validated on real world observations in order to allow for accuracy assessment. Some promising initiatives exist, such as UK NEA, AIRES, INVEST or TEEB. However, most studies still focus either on the spatial distribution of ESS supply or on the spatial distribution of its value per unit of ESS. Only a few studies undertake efforts to incorporate both dimensions in a sophisticated manner. Mapping ESS values is a highly interdisciplinary exercise and requires the integration of ecological and economic research in order to utilise their specific strengths in assessing either the spatial biophysical or socioeconomic dimension of ESS values.

\section{References}

Akter, S., Grafton, R.Q., 2010. 'Confronting Uncertainty and missing values in environmental value transfer as applied to species conservation'. Conservation Biology 24 (5), 1407-1417.

Anderson, B.J., et al., 2009. 'Spatial covariance between biodiversity and other ecosystem service priorities'. Journal of Applied Ecology 46 (4), 888-896.

Armstrong, D.A., Roofer, C., Gunderson, D., 2003. 'Estuarine production of juvenile dungeness crab (cancer magister) and contribution to the Oregon- Washington coastal fishery'. Estuaries 26 (4B), 1174-1188.

Baerenklau, K.A., et al., 2010. 'Spatial Allocation of Forest Recreation Value'. 16 (2), 113-126.

Bateman, I.J., et al., 2002. 'Applying geographical information systems (GIS) to environmental and resource economics'. Environmental and Resource Economics 22 (1), 219-269.

Bateman, I.J., et al., 2011. 'Economic Values from Ecosystems. in UK National Ecosystem Assessment: Understanding Nature's Value to Society, Technical Report'. Cambridge: UNEP-WCMC, p. 1466 (Chapter 22).

Bateman, I.J., et al., 2010. 'Economic analysis for ecosystem service assessments'. Environmental Resource Economics 48 (2), 177-218.

Bateman, I.J., Brainard, J.S., Lovett, A.A., 1995. 'Modelling Woodland Recreation Demand Using Geographical Information Systems: A Benefit Transfer Study'. GEC 95-06.

Bateman, I.J., Jones, A.P., 2003. 'Contrasting conventional with multi-level modelling approaches to meta-analysis: expectation consistency in UK Woodland recreation values'. Land Economics 79 (2), 235-258.

Bateman, I.J., Lovett, A.A., 2000. 'Estimating and valuing the carbon sequestered in softwood and hardwood trees, timber products and forest soils in wales'. Journal of Environmental Management 60 (4), 301-323. 
Bateman, I.J., Lovett, A.A., Brainard, J.S., 1999a. 'Developing a methodology for benefit transfer using geographical information systems: modelling demand for woodland recreation'. Regional Studies 33 (3), 191-205.

Bateman, I.J., Ennew, C., et al., 1999b. 'Modelling and mapping agricultural output values using farm specific details and environmental databases'. Journal of Agricultural Economics 50 (3), 488-511.

Bockstael, N.E., 1996. 'Modelling economics and ecology: the importance of a spatial perspective'. American Journal of Agricultural Economics 78 (5), 1168-1180.

Bockstael, Nancy E., Freeman, A. Myrick, Kopp, J. Raymond, Portney, R. Paul, Smith, V. Kerry, 2000. 'On measuring economic values for nature'. Environmental Science \& Technology 34 (8), 1384-1389.

Brainard, J.S., 1999. 'Integrating geographical information systems into travel cost analysis and benefit transfer'. International Journal of Geographical Information Science 13 (3), 227-246.

Brainard, J.S., Bateman, I.J., Lovett, A.A., 2009b. 'The social value of carbon sequestered in Great Britain's woodlands'. Ecological Economics 68 (4), 1257-1267.

Brander, L.M., et al., 2010. 'Scaling Up Ecosystem Services Values: Methodology, Applicability and a Case Study'. SSRN eLibrary. Available at: /http://papers.ssrn.com/sol3/papers.cfm? abstract_id=1600011S (accessed 21.06.2010).

Brander, L.M., et al., 2011. 'Using meta-analysis and GIS for value transfer and scaling up: valuing climate change induced losses of European wetlands'. Environmental and Resource Economics 52, 395-413.

Brenner, J., et al., 2010. 'An assessment of the non-market value of the ecosystem services provided by the Catalan Coastal Zone, Spain'. Ocean \& Coastal Management 53 (1), 27-38.

Brookshire, D., Chermak, J., Desimone, R., 2007. 'Uncertainty, benefit transfers and physical models: a middle Rio Grande valley focus'. In: Navrud, S., Richard

Ready, Bateman, I.J. (Eds.), 'Environmental Value Transfer: Issues and Methods. The Economics of Non-Market Goods and Resources'. Springer, Netherlands, pp. 89-109.

Brouwer, R., 2000. 'Environmental value transfer: state of the art and future prospects'. Ecological Economics 32, 137-152.

Campbell, D., Hutchinson, W.G., Scarpa, R., 2009. 'Using choice experiments to explore the spatial distribution of willingness to pay for rural landscape improvements'. Environment and PlanningPart A 41 (1), 97-111.

Cardinale, B.J., et al., 2012. 'Biodiversity loss and its impact on humanity'. Nature 486 (7401), 59-67.

Cardinale, B.J., Palmer, M.A., Collins, S.L., 2002. 'Species diversity enhances ecosystem functioning through interspecific facilitation'. Nature 415 (6870), 426-429.

Chan, K., Hoshizaki, L., Klinkenberg, B., 2011. 'Ecosystem services in conservation planning: targeted benefits vs. co-benefits or costs?' PLoS ONE 6 (9), 14.

Chen, N., Li, H., Wang, L., 2009. A GIS-based approach for mapping direct use value of ecosystem services at a county scale: management implications'. Ecological Economics 68 (11), 2768-2776. 
Chiabai, A., et al., 2011. 'Economic assessment of forest ecosystem services losses: cost of policy inaction'. Environmental and Resource Economics 50 (3), 405-445.

Coiner, C., Wu, J., Polasky, S., 2001. 'Economic and environmental implications of alternative landscape designs in the walnut creek watershed of lowa'. Ecological Economics 38 (1), 119-139.

Costanza, R., et al., 2008. 'The value of coastal wetlands for hurricane protection'. Ambio 37 (4), 241248.

Costanza, R., et al., 1997. 'The value of the world's ecosystem services and natural capital'. Nature 387 (6630), 253-260.

Crossman, N.D., et al., 2010. 'Reconfiguring an irrigation landscape to improve provision of ecosystem services'. Ecological Economics 69 (5), 1031-1042.

Crossman, N.D., Bryan, B.A., 2009. 'Identifying cost-effective hotspots for restoring natural capital and enhancing landscape multifunctionality'. Ecological Economics 68 (3), 654-668.

De-yong, Y., et al., 2005. 'Valuation of ecosystem services for Huzhou City, Zhejiang Province from 2001 to 2003 by remote sensing data'. Journal of Forestry Research 16 (3), 223-227.

De Groot, R., et al., 2010. 'Challenges in integrating the concept of ecosystem services and values in landscape planning, management and decision making'. Ecological Complexity 7 (3), 260-272.

De Kok, J.-L., Grossmann, M., 2010. 'Large-scale assessment of flood risk and the effects of mitigation measures along the Elbe river'. Natural Hazards 52 (1), 143-166.

Eade, J.D.O., Moran, D., 1996. 'Spatial economic valuation: benefits transfer using geographical information systems'. Journal of Environmental Management 48 (2), 97-110.

Eigenbrod, F., et al., 2010a. 'Error propagation associated with benefits transfer-based mapping of ecosystem services'. Biological Conservation 143 (11), 2487-2493.

Eigenbrod, F., et al., 2010b. 'The impact of proxy-based methods on mapping the distribution of ecosystem services'. Journal of Applied Ecology 47 (2), 377-385.

Freeman, A.M.I., 2003. 'The Measurement of Environmental and Resource Values'. Resources For The Future, Washington D.C.

Ghermandi, A. et al., 2010. 'Recreational, Cultural and Aesthetic Services from Estuarine and Coastal Ecosystems'. SSRN eLibrary.

Ghermandi, A., Nunes, Paulo A.L.D., 2011. 'A Global Map of Costal Recreation Values: Results from a Spatially Explicit Based Meta-Analysis'. FEEM Working Paper 39.

Gret-Regamey, A., et al., 2008. 'Linking GIS-Based models to value ecosystem services in an alpine region'. Journal of Environmental Management 89 (3), 197-208.

Guo, Z., et al., 2001. 'Ecosystem functions, services and their values-a case study in Xingshan county of China'. Ecological Economics 38 (1), 141-154.

Guo, Z., Xiao, X., Li, D., 2000. 'An assessment of ecosystem services: water flow regulation and hydroelectric power production'. Ecological Applications 10 (3), 925-936.

Hanley, N., Spash, C.L., 1993. 'Cost-Benefit Analysis and the Environment'. Edward Elgar, Vermont. 
Helian, L., et al., 2011. 'Changes in land use and ecosystem service values in Jinan, China'. Energy Procedia 5, 1109-1115.

Holzkämper, A., Seppelt, R., 2007. 'Evaluating cost-effectiveness of conservation management actions in an agricultural landscape on a regional scale'. Biological Conservation 136 (1), 117-127.

Ingraham, M.W., Foster, S.G., 2008. 'The value of ecosystem services provided by the U.S. National Wildlife Refuge System in the Contiguous U.S. '. Ecological Economics 67 (4), 608-618.

Isely, E.S., et al., 2010. 'Addressing the information gaps associated with Valuing Green Infrastructure in West Michigan: integrated valuation of ecosystem services tool (INVEST) '. Journal of Great Lakes Research 36 (3), 448-457.

Jin, Y., Huang, J., Peng, D., 2009. 'A new quantitative model of ecological compensation based on ecosystem capital in Zhejiang Province, China'. Journal of Zhejiang University Science: B 10 (4), 301305.

Johnston, R.J., Rosenberger, R.S., 2010. 'Methods, trends and controversies in contemporary benefit transfer'. Journal of Economic Surveys 24 (3), 479-510.

Konarska, K.M., Sutton, P.C., Castellon, M., 2002. 'Evaluating scale dependence of ecosystem service valuation: a comparison of NOAA-AVHRR and landsat TM datasets'. Ecological Economics 41 (3), 491507.

Kotchen, M.J., Reiling, S.D., 2000. 'Environmental attitudes, motivations, and contingent valuation of nonuse values: a case study involving endangered species'. Ecological Economics 32 (1), 93-107.

Kreuter, U.P., et al., 2001. 'Change in ecosystem service values in the San Antonio Area, Texas'. Ecological Economics 39 (3), 333-346.

Liu, S., et al., 2010. 'Valuing New Jersey's ecosystem services and natural capital: a spatially explicit benefit transfer approach'. Environmental Management 45 (6), 1271-1285.

MA (Millennium Ecosystem Assessment), 2005. 'Ecosystems and Human Well-being: Synthesis'. Island Press, Washington, DC.

Maes, J., Braat, Leon, et al., 2011a. 'A Spatial Assessment of Ecosystem Services in Europe: Methods, Case Studies and Policy Analysis-Phase 1'. Ispra, Italy: Partnership for European Environmental Research.

Maes, J., et al., 2012. 'A Spatial Assessment of Ecosystem Services in Europe: Methods, Case Studies and Policy Analysis-Phase 2'. Ispra, Italy: Partnership for European Environmental Research.

Maes, J., Paracchini, M.L., Zulian, G., 2011b. 'A European Assessment of the Provision of Ecosystem Services-Towards an Atlas of Ecosystem Services'. Luxembourg.

Mashayekhi, Z., et al., 2010. 'Economic valuation of water storage function of forest ecosystems (Case study: Zagros Forests, Iran) '. Journal of Forestry Research 21 (3), 293-300.

McPherson, G., et al., 2011. 'Million Trees Los Angeles Canopy Cover and Benefit Assessment'. Landscape and Urban Planning 99 (1) pp. 40-50.

Moons, E., et al., 2008. 'Optimal location of new forests in a suburban region'. Journal of Forest Economics 14 (1), 5-27. 
Naidoo, R., et al., 2008. 'Global mapping of ecosystem services and conservation priorities'. Proceedings of the National Academy of Sciences 105 (28), 9495-9500.

Naidoo, R., Adamowicz, W.L., 2005. 'Economic benefits of biodiversity exceed costs of conservation at an African rainforest reserve'. Proceedings of the National Academy of Sciences of the United States of America 102 (46), 16712-16716.

Naidoo, R., Adamowicz, W.L., 2006. 'Modelling opportunity costs of conservation in transitional landscapes'. Conservation Biology: The Journal of the Society for Conservation Biology 20 (2), 490500.

Naidoo, R., Ricketts, T.H., 2006. 'Mapping the economic costs and benefits of conservation'. PLoS Biology 4 (11), e360.

Navrud, S., Bergland, O., 2001. In: Spash, C.L., Carter, C. (Eds.), 'Value Transfer and Environmental Policy'. University of Cambridge, Cambridge.

Navrud, S., Ready, R., 2007a. 'Review of methods for value transfer'. In: Navrud, S., Ready, Richard (Eds.), 'Environmental Value Transfer: Issues and Methods'. The Economics of Non-Market Goods and Resources. Springer, Netherlands, pp. 1-10.

Nelson, E.J., et al., 2009. 'Modelling multiple ecosystem services, biodiversity conservation, commodity production, and tradeoffs at landscape scales'. Frontiers in Ecology and the Environment 7 (1), 4-11.

Nelson, E.J., Daily, G.C., 2010. 'Modelling Ecosystem Services in Terrestrial Systems'. F1000 Biology Reports.

Nicholson, E., et al., 2009. 'Priority research areas for ecosystem services in a changing world'. Journal of Applied Ecology 46 (6), 1139-1144.

O'Farrell, P.J., et al., 2011. 'The possibilities and pitfalls presented by a pragmatic approach to ecosystem service valuation in an arid biodiversity hotspot'. Journal of Arid Environments 75 (6), 612-623.

O'Higgins, G., Timothy, G.W., et al., 2010. 'Habitat Scale Mapping of Fisheries Ecosystem Service Values in Estuaries'. Ecology and Society 15 (4), 7.

Pearce, D., Whittington, D., Georgiou, S., James, D., 1994. 'Project and Policy Appraisal: Integrating Economics and Environment. OECD, Paris.

Pearce, D., Atkinson, G., Mourato, S., 2006. 'Cost-Benefit Analysis and the Environment: Recent Developments', Paris, France: OECD Publishing. Available at: / http://eprints.Ise.ac.uk/2867/S (accessed 23.05.2008).

Petrosillo, et al., 2009. 'The effectiveness of different conservation policies on the security of natural capital'. Landscape and Urban Planning 89 (1-2), 49-56.

Petrosillo, I., Semeraro, Teodoro, Zurlini, Giovanni, 2010. 'Detecting the "Conservation effect" on the maintenance of natural capital flow in different natural parks'. Ecological Economics 69 (5), 11151123.

Plummer, M.L., 2009. 'Assessing benefit transfer for the valuation of ecosystem services'. Frontiers in Ecology and the Environment 7 (1), 38-45. 
Polasky, S., et al., 2008. 'Where to put things? Spatial land management to sustain biodiversity and economic returns'. Biological Conservation 141 (6), 1505-1524.

Powe, N.A., et al., 1997. 'Using a geographic information system to estimate a hedonic price model of the benefits of woodland access'. Forestry 70 (2), 139-149.

Pritchard Jr., L., Folke, C., Gunderson, L., 2000. 'Valuation of ecosystem services in institutional context'. Ecosystems 3 (1), 36-40.

Rees, S.E., et al., 2010. 'The value of marine biodiversity to the leisure and recreation industry and its application to marine spatial planning'. Marine Policy 34 (5), 868-875.

Rosenberger, R.S., Johnston, R.J., 2009. 'Selection effects in meta-analysis and benefit transfer: avoiding unintended consequences'. Land Economics 85 (3), 410-428.

Rosenberger, R.S., Phipps, T., 2007. 'Correspondence and convergence in benefit transfer accuracy: meta-analytic review of the literature'. In: Navrud, S., Ready, Richard (Eds.), Environmental Value Transfer: Issues and Methods. Dordrecht. Springer, Netherlands, pp. 23-43.

Sagoff, M., 2004. 'Price, Principle, and the Environment'. Cambridge University Press, Cambridge.

Sandhu, H.S., et al., 2008. 'The future of farming: the value of ecosystem services in conventional and organic arable land'. An experimental approach. Ecological Economics 64 (4), 835-848.

Scheurle, C., Thebault, H., Duffa, C., 2010. 'Towards a decision support tool: sensitivity mapping of the French Mediterranean Coastal environment (a case study of fishery and lodging) '. In: Aravossis, K., Brebbia, C.A., (Eds.), 'Environmental Economics and Investment Assessment III'. WIT Press, Southampton, pp. 135-145.

Seidl, A.F., Moraes, A.S., 2000. 'Global valuation of ecosystem services: application to the Pantanal da Nhecolandia, Brazil'. Ecological Economics 33 (1), 1-6.

Simonit, S., Perrings, C., 2011. 'Sustainability and the value of the "regulating" services: wetlands and water quality in Lake Victoria'. Ecological Economics 70 (6), 1189-1199.

Spash, C.L., Carter, C., 2001. 'Environmental Valuation in Europe: Findings from the Concerted Action'. Cambridge Research for the Environment, Department of Land Economy, University of Cambridge.

Spash, C.L., Vant, A., 2006. 'Transferring environmental value estimates: issues and alternatives'. Ecological Economics 60 (2), 379-388.

Stanley, T.D., Rosenberger, R.S., 2009. 'Are Recreation Values Systematically Underestimated? Reducing Publication Selection Bias for Benefit Transfer'. Working Paper. Conway, AR: Department of Economics, Hendrix College.

Sutton, P.C., Costanza, R., 2002. 'Global estimates of market and non-market values derived from night time satellite imagery, land cover, and ecosystem service valuation'. Ecological Economics 41 (3), 509-527.

Tallis, H., Polasky, S., 2009. 'Mapping and valuing ecosystem services as an approach for conservation and natural-resource management'. Annals of the New York Academy of Sciences 1162, 265-283. 
TEEB (The Economics of Ecosystems \& Biodiversity), 2010. 'The Economics of Ecosystems and Biodiversity: Ecological and Economic Foundations' London. Earthscan, Washington D.C..

Termansen, M., Zandersen, M., McClean, C.J., 2008. 'Spatial substitution patterns in forest recreation'. Regional Science and Urban Economics 38 (1), 81-97.

Troy, A., Wilson, M.A., 2006. 'Mapping ecosystem services: practical challenges and opportunities in linking GIS and value transfer'. Ecological Economics 60 (2), 435-449.

UK NEA, 2011. 'UK National Ecosystem Assessment: Understanding Nature's Value to SocietySynthesis of the Key Findings'. Cambridge.

Viglizzo, E.F., Frank, F.C., 2006. 'Land-use options for Del Plata Basin in South America: tradeoffs analysis based on ecosystem service provision'. Ecological Economics 57 (1), 140-151.

Wei, G., et al., 2007. 'Comparison of changes of typical river segment ecosystem service value in LRGR'. Chinese Science Bulletin 52, 262-272.

Williams, E., et al., 2003. 'The value of Scotland's ecosystem services and natural capital'. European Environment 13 (2), 67-78.

Yoshida, A., et al., 2010. 'Ecosystem service values land and use change in the opium poppy cultivation region in northern part of Lao PDR'. Acta Ecologica Sinica 30 (2), 56-61.

Yu, et al., 2005. 'Grassland ecosystem services and their economic evaluation in Qinghai-Tibetan plateau based on RS and GIS'. IGARSS 2005. In: 'IEEE International Geoscience and Remote Sensing Symposium Proceedings', Vol. 18, pp. 0-2961.

Yuan, L., et al., 2006. 'Land use change and its impact on values of ecosystem services in the West of Jilin Province'. Wuhan University Journal of Natural Sciences 11 (4), 1028-1034.

Zandersen, M., Tol, R.S.J., 2009. 'A meta-analysis of forest recreation values in Europe'. Journal of Forest Economics 15 (1-2), 109-130.

Zhang, Jingcheng, et al., 2011a. 'An ecological based sustainability assessing system for cropping system'. Mathematical and Computer Modelling 54 (3-4), 1160-1166.

Zhang, M., et al., 2011b. 'Spatiotemporal variation of karst ecosystem service values and its correlation with environmental factors in Northwest Guangxi, China'. Environmental Management 48 (5), 933-944.

Zhang, W., et al., 2007. 'Assessment of land use change and potential eco-service value in the upper reaches of Minjiang River, China'. Journal of Forestry Research 18 (2), 97-102.

Zhao, B., et al., 2004. 'An ecosystem service value assessment of land-use change on Chongming Island, China'. Land Use Policy 21 (2), 139-148.

Zhao, B., et al., 2005. 'Estimation of ecological service values of wetlands in Shanghai, China'. Chinese Geographical Science 15 (2), 151-156.

Zhiyuan, R., Yanfang, Z., Jing, L., 2003. 'The value of vegetation ecosystem services: a case of QinlingDaba Mountains'. Journal of Geographical Sciences 13 (2), 195-200. 